\title{
Homotopy invariants of higher dimensional categories and concurrency in computer science
}

\author{
Philippe Gaucher \\ Institut de Recherche Mathématique Avancée \\ U.L.P. et C.N.R.S. \\ 7 rue René Descartes \\ 67084 Strasbourg Cedex \\ France \\ email : gaucher@irma.u-strasbg.fr
}

December 1999

\begin{abstract}
The strict globular $\omega$-categories formalize the execution paths of a parallel automaton and the homotopies between them. One associates to such (and any) $\omega$-category $\mathcal{C}$ three homology theories. The first one is called the globular homology. It contains the oriented loops of $\mathcal{C}$. The two other ones are called the negative (resp. positive) corner homology. They contain in a certain manner the branching areas of execution paths or negative corners (resp. the merging areas of execution paths or positive corners) of $\mathcal{C}$. Two natural linear maps called the negative (resp. the positive ) Hurewicz morphism from the globular homology to the negative (resp. positive) corner homology are constructed. We explain the reason why these constructions allow the reinterpretation of some geometric problems coming from computer science.
\end{abstract}

\section{Contents}

\begin{tabular}{|lll}
\hline 1 & Introduction & 3
\end{tabular}

2 Presentation of the geometric ideas of this work 4

$2.1 \quad$ Execution paths and homotopies between them in a very informal way . . . 4

2.2 The free $\omega$-category generated by a cubical set $\ldots \ldots \ldots$. . . . . 8

$2.3 \quad \omega$-categories up to homotopy $\ldots \ldots \ldots \ldots \ldots$ 
3 Globular homology of $\omega$-category 11

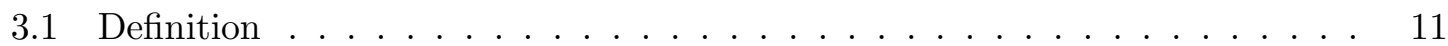

3.2 Functorial property of the globular homology . . . . . . . . . . . . . . 12

3.3 Homological property $\ldots \ldots \ldots \ldots \ldots \ldots$

\begin{tabular}{|lll}
4 & Positive and negative corner homology of an $\omega$-category & 16
\end{tabular}

4.1 The pasting scheme $\Lambda^{n}$ and the $\omega$-category $I^{n} \ldots \ldots \ldots \ldots$

4.2 The cubical singular nerve . . . . . . . . . . . . . . . . . . . 18

4.3 Construction of the corner homologies . . . . . . . . . . . . . . . . 20

4.4 Examples of corners . . . . . . . . . . . . . . . . . . 21

5 Filling of shells in the cubical singular nerve 22

5.1 Recall about the freeness of $I^{n} \ldots \ldots \ldots \ldots . \ldots \ldots 22$

$5.2 \quad$ Filling of shells using the freeness of $I^{n} \ldots \ldots \ldots \ldots$. . . . . . . . 23

6 Two new simplicial nerves 25

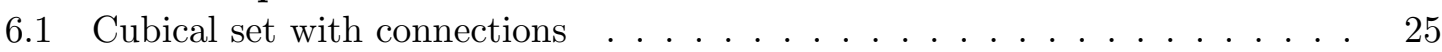

6.2 Construction of connections on the cubical singular nerve . . . . . . . . . 26

\begin{tabular}{|lll}
\hline 7 & The oriented Hurewicz morphisms & 28
\end{tabular}

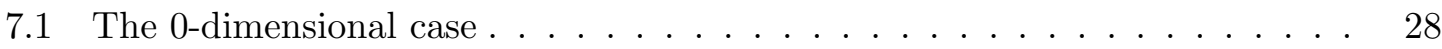

7.2 The 1-dimensional case . . . . . . . . . . . . . . . . . . . . . 28

7.3 The higher dimensional case $\ldots \ldots \ldots \ldots$. . . . . . . . . . . . . 29

8 Toward an "oriented algebraic topology" 33

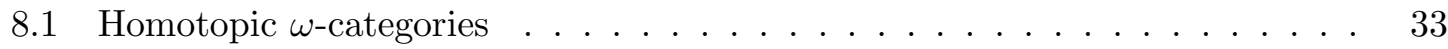

8.2 Invariance of the globular and corner homologies $\ldots \ldots \ldots$. . . . . . . . 39

9 Some open questions and perspectives 40

9.1 Some interesting problems in mathematics . . . . . . . . . . . . . . . . . 40

9.2 Perspectives in computer science $\ldots \ldots \ldots \ldots \ldots$. . . . . . . . . . 43

10 Direct construction of the globular and corner homologies of a cubical $\begin{array}{ll}\text { set } & 44\end{array}$

10.1 Cubical $\omega$-category $\ldots \ldots \ldots \ldots \ldots$. . . . . . . . . . . . . 44

10.2 The globular and corner homologies of a cubical set $\ldots \ldots$. . . . . . 46

\begin{tabular}{ll}
\hline 1 Acknowledgments & 50
\end{tabular}

12 The categories and functors of this work 51 


\section{Introduction}

The use of geometric notions to describe the behaviour of concurrent machines is certainly not new since progress graphs [Dij68], HDA Pra91] [vG91] and simplicial models of [HS94] [HR94. The purpose of this article is to provide a new setting for the homotopy of execution paths in concurrent automata, in order to improve the homological approach of Gou95. We can point out that some other approaches of this question already exist. With partially ordered topological spaces in FGR98a. And with partial posets in [Sok99]. Pratt already noticed that a good structure to deal with execution paths and homotopies between them is the structure of globular $\omega$-category Pra91. The aim of this paper is threefold. First the use of the concept of globular $\omega$-category to describe concurrent machines is justified on some well-known examples and in a very informal way. Secondly we associate to every globular $\omega$-category three homology theories and two natural maps between them. We explain why their content is interesting for some geometric problems coming from computer science. Thirdly, as in algebraic topology, a notion of homotopic $\omega$-categories is proposed and we prove that the preceding homology theories are invariant with respect to it (only in a particular case for the corner homologies).

Now here is the organization of the paper. In Section 2, we make precise the notion of paths and homotopies between paths, homotopies between homotopies, etc... The notion of globular $\omega$-category is recalled. The link between the usual formalization of concurrent automata using cubical sets and the new formalization by globular $\omega$-categories is explained. We give in a very informal way the geometric and computer science meaning of the homology theories which will be constructed in this paper. In Section 3, the globular homology of a globular $\omega$-category is defined. Some examples of globular cycles are given and the globular complex is related to a derived functor. Section 4 is devoted to the construction of the negative and positive corner homologies of an $\omega$-category. Afterwards we introduce in Section 5 a technical tool to fill shells in the cubical singular nerve of an $\omega$-category. Next in Section 6, this notion of filling of shell is used to construct two families of connections on the cubical singular nerve of an $\omega$-category. It allows us to prove that the corner homologies are the homologies associated to two new simplicial nerves. Afterwards we construct in Section 7 the two natural maps from the globular homology to the negative and positive corner homologies. In Section 8, a notion of homotopy equivalence of $\omega-$ categories is proposed. We will prove the following property : Let $f$ and $g$ be two non 1 -contracting $\omega$-functors from $\mathcal{C}$ to $\mathcal{D}$. If $f$ and $g$ are homotopic, then for any natural

number $n, H_{n}^{g l}(f)=H_{n}^{g l}(g)$ (Theorem 8.6). In a very particular case, it is also possible to relate the homotopy of paths in $\omega$-categories to the corner homology theories $H_{n}^{-}$and $H_{n}^{+}$ (Theorem 8.7). In Section 9, some conjectures and perspectives both in mathematics and computer science are exposed. In Section 10, we prove that the cubical singular nerve of the free $\omega$-category generated by a cubical set $K$ is nothing else but the free cubical $\omega$-category generated by $K$. It allows us to propose a direct construction of the globular homology and 
of the corner homologies of a cubical set without using any globular $\omega$-category.

\section{Presentation of the geometric ideas of this work}

\subsection{Execution paths and homotopies between them in a very informal way}

A sequential machine (i.e. without concurrency) is a set of states, also called 0-transitions, and a set of 1-transitions from a given state to another one. A concurrent machine, like the previous one, consists of a set of states and a set of 1-transitions but has also the capability of carrying out several 1-transitions at the same time.

In Figure 1, if we work in cartesian coordinates in such a way that $A=[0,1] \times[0,1]$ with $\alpha=(0,0)$ and $\delta=(1,1)$, the set of continuous maps $\left(c_{1}, c_{2}\right)$ from $[0,1]$ to $A$ such that $c_{1}(0)=c_{2}(0)=0, c_{1}(1)=c_{2}(1)=1$ and $t \leqslant t^{\prime}$ implies $c_{1}(t) \leqslant c_{1}\left(t^{\prime}\right)$ and $c_{2}(t) \leqslant c_{2}\left(t^{\prime}\right)$ represents all the simultaneous possible executions of $u$ and $v$. Coordinates represent the evolution of $u$ and $v$, that means the local time taken to execute $u$ or $v$. If $\left(c_{1}, c_{2}\right)(] 0,1[)$ is entirely included in the interior of $A$, it is a "true parallelism". If $\left(c_{1}, c_{2}\right)(] 0,1[)$ is entirely included in the edge of the square, that means that $u$ and $v$ are sequentially carried out by the automaton. More generally, the concurrent execution of $n 1$-transitions can be represented by a $n$-cube. This is already noticed for example in [Pra91] and [Gou95].

Definition 2.1. A cubical set consists of a family of sets $\left(K_{n}\right)_{n \geqslant 0}$, of a family of face maps $K_{n} \stackrel{\partial_{i}^{\alpha}}{\longrightarrow} K_{n-1}$ for $\alpha \in\{-,+\}$ and of a family of degeneracy maps $K_{n-1} \stackrel{\epsilon_{i}}{\longrightarrow} K_{n}$ with $1 \leqslant i \leqslant n$ which satisfy the following relations

1. $\partial_{i}^{\alpha} \partial_{j}^{\beta}=\partial_{j-1}^{\beta} \partial_{i}^{\alpha}$ for all $i<j \leqslant n$ and $\alpha, \beta \in\{-,+\}$

2. $\epsilon_{i} \epsilon_{j}=\epsilon_{j+1} \epsilon_{i}$ for all $i \leqslant j \leqslant n$

3. $\partial_{i}^{\alpha} \epsilon_{j}=\epsilon_{j-1} \partial_{i}^{\alpha}$ for $i<j \leqslant n$ and $\alpha \in\{-,+\}$

4. $\partial_{i}^{\alpha} \epsilon_{j}=\epsilon_{j} \partial_{i-1}^{\alpha}$ for $i>j \leqslant n$ and $\alpha \in\{-,+\}$

5. $\partial_{i}^{\alpha} \epsilon_{i}=I d$

The corresponding category of cubical sets, with an obvious definition of its morphisms, is isomorphic to the category of presheaves Sets ${ }^{\square^{o p}}$ over a small category $\square$. This latter can be described in a nice way as follows Cra95. The objects of $\square$ are the sets $\underline{n}=\{1, \ldots, n\}$ where $n$ is a natural number greater or equal than 1 and an arrow $f$ from $\underline{n}$ to $\underline{m}$ is a function $f^{*}$ from $\underline{m}$ to $\underline{n} \cup\{-,+\}$ such that $f^{*}(k) \leqslant f^{*}\left(k^{\prime}\right) \in \underline{n}$ implies $k \leqslant k^{\prime}$ and $f^{*}(k)=f^{*}\left(k^{\prime}\right) \in \underline{n}$ implies $k=k^{\prime}$. 


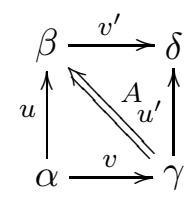

Figure 1: A 2-transition

Let $X$ be a topological space. Let $[0,1]$ denote the interval between 0 and 1 . Set $K_{n}=C^{0}\left([0,1]^{n}, X\right)$ the set of continuous maps from the $n$-box $[0,1]^{n}$ to $X$. Set

$$
\begin{aligned}
& \partial_{i}^{-}(f)\left(x_{1}, \ldots, x_{p}\right)=f\left(x_{1}, \ldots,[0]_{i}, \ldots, x_{p}\right) \\
& \partial_{i}^{+}(f)\left(x_{1}, \ldots, x_{p}\right)=f\left(x_{1}, \ldots,[1]_{i}, \ldots, x_{p}\right) \\
& \epsilon_{i}(f)\left(x_{1}, \ldots, x_{p}\right)=f\left(x_{1}, \ldots, \widehat{x}_{i}, \ldots, x_{p}\right)
\end{aligned}
$$

Then we obtain a cubical set $K$ which is called the cubical singular nerve of the topological space $X$.

In Figure 1, we call $A$ an homotopy between the two 1-paths $u v^{\prime}$ and $v u^{\prime}$. We call 2-path an homotopy between two 1 -paths and by induction on $n \geqslant 2$, we call $n$-path an homotopy between two $(n-1)$-paths. This notion of homotopy is different from the classical one in the sense that only the 1-paths $u v^{\prime}$ and $v u^{\prime}$ are homotopic and because the 1-paths are oriented. For example, still in Figure 1, $u$ is homotopic neither with $u^{\prime}$ nor with $v$ or $v^{\prime}$.

In Figure 2, there are an initial state $\alpha$, a final state $\beta$, two 1-transitions $u$ and $v$ and two 2-transitions or homotopies $A$ and $B$ between $u$ and $v$. Choosing an orientation for $A$ and $B$, for example $s_{1} A=s_{1} B=u$ and $t_{1} A=t_{1} B=v$ ( $s$ for source and $t$ for target), we see that $s_{0} s_{1} A=s_{0} t_{1} A=\alpha$ and $t_{0} s_{1} A=t_{0} t_{1} A=\beta$. These are precisely the globular equations which appear in the axioms of globular $\omega$-categories.

The concatenation yields an associative composition law on the set of 1-transitions of an $\omega$-category. It turns out that there is also a natural composition law on the set of 2transitions. In Figure 3, with the convention of orientation $t_{1} A=s_{1} B$, we can compose $A$ and $B$. Denote this composition by $A *_{1} B$. We see that $s_{1}\left(A *_{1} B\right)=s_{1} A, t_{1}\left(A *_{1} B\right)=$ $t_{1} B$. The composition of higher dimensional morphisms must be associative because it corresponds to the concatenation of the real execution paths contained in $A$ and $B$.

Thus the geometric properties of transitions of concurrent machines can be encoded in a structure of cubical set. And their associated set of execution paths and homotopies between them have a natural structure of globular $\omega$-category. All these ideas already appear in Pra91. Pratt uses the term of $n$-complex which is in fact nothing else but a small $n$-category. We use the notations of [Ste91] and Str87 for the following definition. 


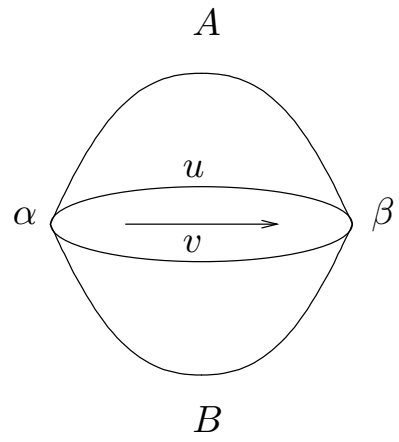

Figure 2: A 3-dimensional hole

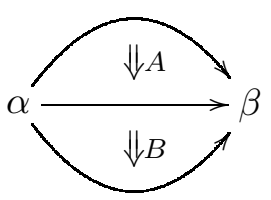

Figure 3: Composition of two 2-morphims 
The following definition already appears in BH81a]

Definition 2.2. An $\omega$-category is a set $A$ endowed with two families of maps $\left(s_{n}=d_{n}^{-}\right)_{n \geqslant 0}$ and $\left(t_{n}=d_{n}^{+}\right)_{n \geqslant 0}$ from $A$ to $A$ and with a family of partially defined 2-ary operations $\left(*_{n}\right)_{n \geqslant 0}$ where for any $n \geqslant 0, *_{n}$ is a map from $\left\{(a, b) \in A \times A, t_{n}(a)=s_{n}(b)\right\}$ to $A((a, b)$ being carried over $\left.a *_{n} b\right)$ which satisfies the following axioms for all $\alpha$ and $\beta$ in $\{-,+\}$ :

1. $d_{m}^{\beta} d_{n}^{\alpha} x=\left\{\begin{array}{l}d_{m}^{\beta} x \text { if } m<n \\ d_{n}^{\alpha} x \text { if } m \geqslant n\end{array}\right.$

2. $s_{n} x *_{n} x=x *_{n} t_{n} x=x$

3. if $x *_{n} y$ is well-defined, then $s_{n}\left(x *_{n} y\right)=s_{n} x, t_{n}\left(x *_{n} y\right)=t_{n} y$ and for $m \neq n$, $d_{m}^{\alpha}\left(x *_{n} y\right)=d_{m}^{\alpha} x *_{n} d_{m}^{\alpha} y$

4. as soon as the two members of the following equality exist, then $\left(x *_{n} y\right) *_{n} z=$ $x *_{n}\left(y *_{n} z\right)$

5. if $m \neq n$ and if the two members of the equality make sense, then $\left(x *_{n} y\right) *_{m}\left(z *_{n} w\right)=$ $\left(x *_{m} z\right) *_{n}\left(y *_{m} w\right)$

6. for any $x$ in $A$, there exists a natural number $n$ such that $s_{n} x=t_{n} x=x$ (the smallest of these numbers is called the dimension of $x$ and is denoted by $\operatorname{dim}(x)$ ).

We will sometimes use the notations $d_{n}^{-}:=s_{n}$ and $d_{n}^{+}=t_{n}$. If $x$ is a morphism of an $\omega$-category $\mathcal{C}$, we call $s_{n}(x)$ the $n$-source of $x$ and $t_{n}(x)$ the $n$-target of $x$. The category of all $\omega$-categories (with the obvious morphisms) is denoted by $\omega$ Cat. The corresponding morphisms are called $\omega$-functors.

If $S$ is a set, the free abelian group generated by $S$ is denoted by $\mathbb{Z} S$. By definition, an element of $\mathbb{Z} S$ is a formal linear combination of elements of $S$.

Definition 2.3. Let $\mathcal{C}$ be an $\omega$-category. Let $\mathcal{C}_{n}$ be the set of $n$-dimensional morphisms of $\mathcal{C}$. Two $n$-morphisms $x$ and $y$ are homotopic if there exists $z \in \mathbb{Z C}_{n+1}$ such that $s_{n} z-t_{n} z=$ $x-y$. This property is denoted by $x \sim y$.

If $x \sim y$, then the pair $(x, y)$ belongs to the reflexive, symmetric and transitive closure of the binary relation generated by all pairs $\left(s_{n}(u), t_{n}(u)\right)$ where $u$ runs over $\mathcal{C}_{n+1}$.

Figure 1 is a very simple example of a distributed database. The hole in the middle corresponds to a mutual exclusion. See [FGR98b] for a complete treatment. The two 1paths $\gamma_{1}$ and $\gamma_{2}$ are homotopic because there exists a 2-morphism between $\gamma_{1}$ and $\gamma_{3}$ and another one between $\gamma_{2}$ and $\gamma_{3}$. On the other hand none of the previous three 1-paths is homotopic to $\gamma_{4}$ because of the oriented hole in the middle. 


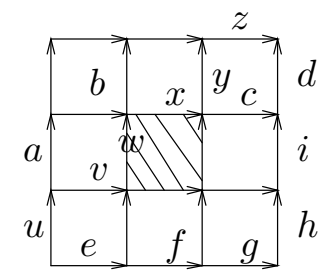

$$
\begin{aligned}
& \gamma_{1}=u *_{0} v *_{0} w *_{0} x *_{0} y *_{0} z \\
& \gamma_{2}=u *_{0} a *_{0} b *_{0} x *_{0} c *_{0} d \\
& \gamma_{3}=u *_{0} v *_{0} w *_{0} x *_{0} c *_{0} d \\
& \gamma_{4}=e *_{0} f *_{0} g *_{0} h *_{0} i *_{0} d
\end{aligned}
$$

Figure 4: Example of distributed database

\subsection{The free $\omega$-category generated by a cubical set}

How may we mathematically associate to every cubical set $K$ its corresponding set of execution paths and higher dimensional homotopies? The link between the two formalizations is as follows. We need to describe precisely the free $\omega$-category associated to each $n$-cube of $K$. In a very informal way, it consists of seeing the faces of a $n$-cube as words of length $n$ in the alphabet $\{-, 0,+\}$. The term $0_{n}$ means $0 n$ times, i.e. the interior of the $n$-cube. And we say that the face $k_{1} \ldots k_{n}$ is at the source of $x$ if $k_{i} \neq 0$ implies $k_{i}=(-1)^{i}$ and we say that $k_{1} \ldots k_{n}$ is at the target of $x$ if $k_{i} \neq 0$ implies $k_{i}=(-1)^{i+1}$. We will make precise the construction of $I^{n}$ in Section 4.1. Once this is done, it suffices to paste the $\omega$-categories associated to every $n$-cube of $K$ in the same way that they are pasted in $K$. More concretely, every cubical set $K$ is in a canonical way the direct limit of the elementary $n$-cubes included in it. This is due to the fact that any functor from a small category to the category Sets of sets is a canonical direct limit of representable functors, the set of those functors being dense in the set of all set-valued functors. More precisely, we have $K=\int^{\underline{n} \in \square} K_{n} . \square(-, \underline{n})$ where the integral sign is the coend construction [ML71] and $K_{n} \cdot \square(-, \underline{n})$ means the sum of "cardinal of $K_{n}$ " copies of $\square(-, \underline{n})$. So $F(K)=\int^{\underline{n} \in \square} K_{n} \cdot I^{n}$ is a $\omega$-category containing as 1-morphisms all arrows of $K$ and all possible compositions of these arrows, as 2-morphisms all homotopies between the execution paths, etc...

Consider for example the 2-cube of Figure 5. The 2-face 00 is oriented from the side $\{-0,0+\}$ to the side $\{0-,+0\}$. The corresponding $\omega$-category $I^{2}$ contains all possible compositions of faces of the 2-cube. Therefore, as set, we have

$$
I^{2}=\left\{--,-+,+-,++,-0,0-,+0,0+,-0 *_{0} 0+, 0-*_{0}+0,00\right\} .
$$

In Figure 6, the three 2-morphisms $A, B$ and $C$ are not drawn and are supposed to be 


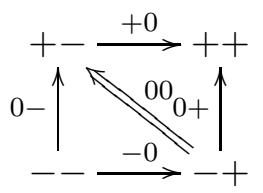

Figure 5: The $\omega$-category $I^{2}$

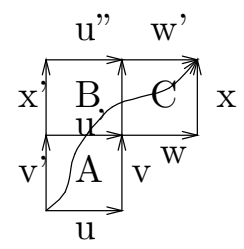

$\left(A *_{0} w *_{0} x\right) *_{1}\left(v^{\prime} *_{0}\left(\left(u^{\prime} *_{0} C\right) *_{1}\left(B *_{0} w^{\prime}\right)\right)\right)$

Figure 6: Composition of three squares

oriented to the north west. The composition of the three squares $A, B$ and $C$ is equal to

$$
\left(A *_{0} w *_{0} x\right) *_{1}\left(v^{\prime} *_{0}\left(\left(u^{\prime} *_{0} C\right) *_{1}\left(B *_{0} w^{\prime}\right)\right)\right)
$$

in the free $\omega$-category generated by this cubical set.

The map $F$ induces a functor from the category of cubical sets to the category of $\omega$ categories. Indeed this is the left Kan extension of the functor $Q$ from $\square$ to $\omega$ Cat defined as follows ML71. It maps $\underline{n}$ to $I^{n}$. Let $\epsilon_{i}$ be the surjective morphism from $\underline{n}$ to $\underline{n-1}$ for $1 \leqslant i \leqslant n$ defined by $\left(\epsilon_{i}\right)^{*}(l)=l$ if $l<i$ and $\left(\epsilon_{i}\right)^{*}(l)=l+1$. Let $\partial_{i}^{\alpha}$ be the injective morphism from $\underline{n-1}$ to $\underline{n}$ for $1 \leqslant i \leqslant n$ and for $\alpha= \pm$ defined by $\left(\partial_{i}^{\alpha}\right)^{*}(l)=l$ if $l<i$, $\left(\partial_{i}^{\alpha}\right)^{*}(l)=\alpha$ for $l=i$ and $\left(\partial_{i}^{\alpha}\right)^{*}(l)=l-1$ for $l>i$. Then any morphism of $\square$ is a composition of $\epsilon_{i}$ and of $\partial_{i}^{\alpha}$. And $Q$ is the unique functor which maps $\epsilon_{i}$ to $\epsilon_{i}$ and $\partial_{i}^{\alpha}$ to $\partial_{i}^{\alpha}$ Cra95. This way, the notion of $\omega$-category can be understood as a generalization of the notion of cubical set. Every cubical set can be seen as an $\omega$-category. The converse is false. We will see in Section 8 why this categoric setting is very well adapted for the development of an analogue of algebraic topology in the computer science framework.

\section{$2.3 \quad \omega$-categories up to homotopy}

Now we want to give, in a very informal way, examples of $\omega$-categories which have the same set of execution paths up to homotopy and to explain the potential interest of this notion. We will propose a definition of homotopic $\omega$-categories in Definition 8.1 and 8.2 . 


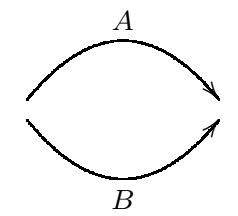

Figure 7: The oriented globe $G_{1}$
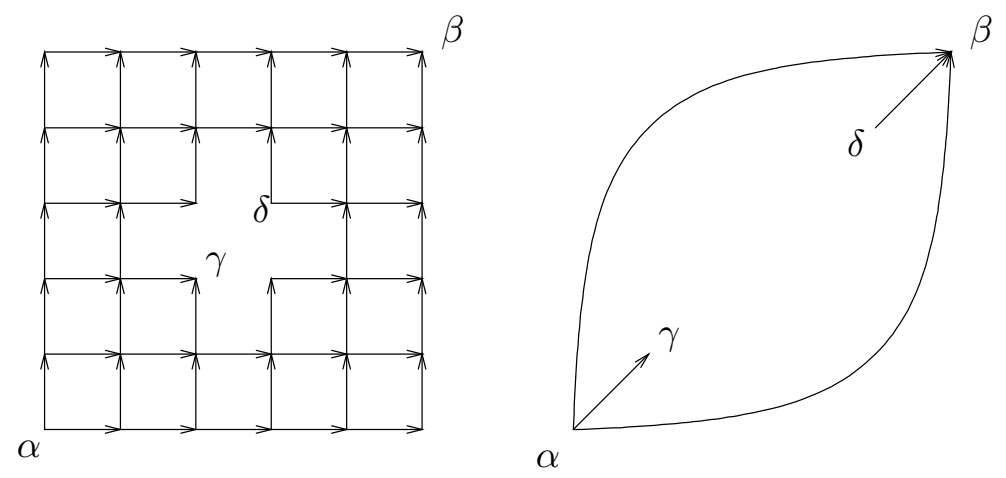

Figure 8: The Swiss Flag

Up to path homotopy, the $\omega$-category of Figure 4 must be the same as the $\omega$-category of Figure 7 because there are only two execution paths up to homotopy in each case.

In Figure 8, the left side is the Swiss Flag example. It is again an example of cubical set appearing in the theory of distributed databases as explained in [FGR98b. The globular $\omega$-category on the right side should be homotopic to the left one.

Our claim is that the most interesting computer-scientific properties of two concurrent machines are the same if the corresponding globular $\omega$-categories are homotopy equivalent. In Figure 8, the state $\gamma$ corresponds to a deadlock of the corresponding concurrent machine. The deadlock appears also on the right. Compare the number of possible execution paths on the left and the only four execution paths on the right which are essentially the same ! This means that an algorithm which could be able to take in account this notion of $\omega$-category up to homotopy would be more efficient that any other algorithm.

Instead of dealing directly with $\omega$-category up to homotopy, a more fruitful approach consists of building some functors from $\omega$-categories to, for example, abelian groups, invariant up to homotopy. These functors contain, at least theoretically, a relevant geometric information because of their invariance up to homotopy in the above sense. A usual way to construct such invariants consists of constructing functors from the category of $\omega$-categories 
to the category $\operatorname{Comp}(A b)$ of chain complexes of groups and to consider the associated homology groups.

Definition 2.4. A chain complex of groups is a family of abelian groups $\left(C_{n}\right)_{n \geqslant 0}$ together with a family of linear maps $\partial_{n}: C_{n+1} \longrightarrow C_{n}$ such that $\partial_{n} \circ \partial_{n+1}=0$ for any $n \geqslant 0$.

Since the image of $\partial_{n+1}$ is included in the kernel of $\partial_{n}$, the quotient group

$$
H_{n}\left(C_{*}, \partial_{*}\right)=\operatorname{Ker}\left(\partial_{n}\right) / \operatorname{Im}\left(\partial_{n+1}\right)
$$

is well defined. It is called the $n$-th homology group of the group complex $\left(C_{n}\right)_{n \geqslant 0}$. The map $H_{n}$ yields a functor from $\operatorname{Comp}(A b)$ to the category $A b$ of abelian groups. See for example [Rot79] or Wei94 for an introduction to the theory of these mathematical objects.

The first homology theory will be the globular homology $H_{*}^{g l}(\mathcal{C})$ (Definition 3.1). An example of globular cycle of dimension 1 is $\gamma_{1}-\gamma_{4}$ of Figure 1 . We call it an oriented 1-dimensional loop. An example of globular cycle of dimension 2 is $A-B$ of Figure 2.

The two other homology theories will be called the negative and positive corner homologies $H_{*}^{ \pm}(\mathcal{C})$ (Definition 4.3). The cycles of the negative one correspond to the branching areas of execution paths (or negative corner) and the positive one to the merging areas of execution paths (or positive corner). In the case of $\gamma_{1}-\gamma_{4}$ of Figure 4, there is one branching area on the left and one merging area on the right. Idem for Figure 2 .

The idea afterwards is to associate to any oriented loop of any dimension its corresponding negative or positive corners. This is the underlying geometric meaning of the morphisms $h_{*}^{ \pm}$from $H_{*}^{g l}(\mathcal{C})$ to $H_{*}^{ \pm}(\mathcal{C})$ (Proposition 7.5 and 7.7). We can immediately see an application of these maps. Looking back to the Swiss Flag example of Figure 8, it is clear that the cokernel of $h_{1}^{-}$is not empty, because of the deadlock and the forbidden area. A negative corner which yields a non trivial element in this cokernel is drawn in Figure 9 . In the same way, the cokernel of $h_{1}^{+}$in the Swiss Flag example is still not empty, because of the unreachable state and the unreachable area. A positive corner which yields a non zero element of this cokernel is represented in Figure 9.

These geometric remarks ensure that the homology groups that we are going to construct contain relevant information about the geometry of concurrency.

\section{Globular homology of $\omega$-category}

\subsection{Definition}

The starting point is an $\omega$-category $\mathcal{C}$.

Definition 3.1. Let $\left(C_{*}^{g l}(\mathcal{C}), \partial^{g l}\right)$ be the chain complex defined as follows : $C_{0}^{g l}(\mathcal{C})=\mathbb{Z} \mathcal{C}_{0} \oplus$ $\mathbb{Z} \mathcal{C}_{0}$, and for $n \geqslant 1, C_{n}^{g l}(\mathcal{C})=\mathbb{Z C}_{n}, \partial^{g l}(x)=\left(s_{0} x, t_{0} x\right)$ if $x \in \mathbb{Z C}_{1}$ and for $n \geqslant 1, x \in \mathbb{Z} \mathcal{C}_{n+1}$ 


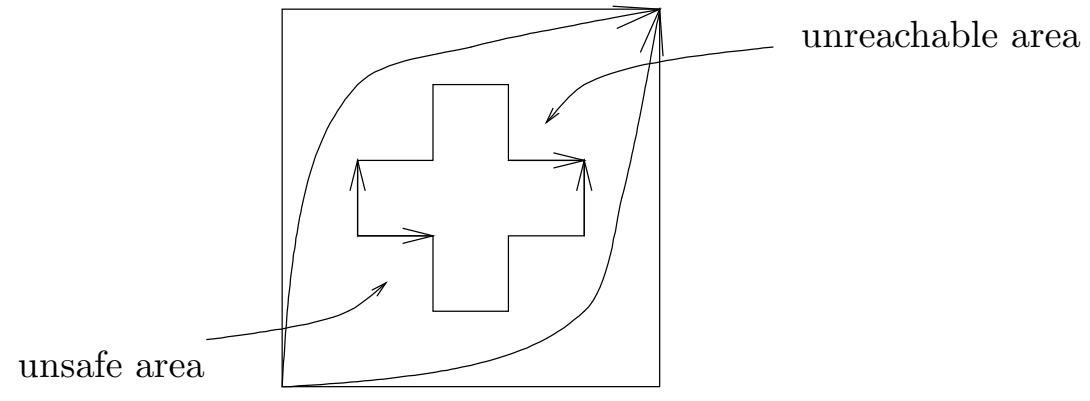

Figure 9: Unsafe area and unreachable area in a concurrent machine with semaphores

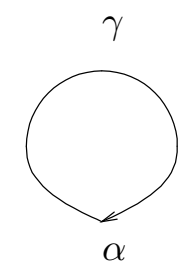

Figure 10: A loop which does not give rise to a globular cycle

implies $\partial^{g l}(x)=s_{n} x-t_{n} x$. This complex is called the globular complex of $\mathcal{C}$ and its corresponding homology the globular homology.

There is a difference between the 1-dimensional case and the other cases. A loop as in Figure 10 where $\gamma$ is a 1-morphism such that $s_{0} \gamma=t_{0} \gamma=\alpha$ does not yield a globular 1 -cycle. However a loop as in Figure 11 where $A$ is a $n$-morphism with $n \geqslant 2$ and such that $s_{n-1} A=t_{n-1} A=\gamma$ yields a globular $n$-cycle.

\subsection{Functorial property of the globular homology}

Now an important technical definition for the sequel.

Definition 3.2. Let $f$ be an $\omega$-functor from $\mathcal{C}$ to $\mathcal{D}$. The morphism $f$ is non 1-contracting if for any 1-dimensional $x \in \mathcal{C}$, the morphism $f(x)$ is a 1-dimensional morphism of $\mathcal{D}$.

The category of $\omega$-categories with the non 1 -contracting $\omega$-functors is denoted by $\omega C_{1} t_{1}$. The category of cubical sets equipped with the non 1-contracting morphisms is denoted by Sets ${ }_{1}^{\square \text { op }}$.

If $f$ is a non 1-contracting $\omega$-functor from $\mathcal{C}$ to $\mathcal{D}$, then for any morphism $x \in \mathcal{C}$ of dimension greater than $1, f(x)$ is of dimension greater than one as well. This is due to the equality $f\left(s_{1} x\right)=s_{1} f(x)$. 


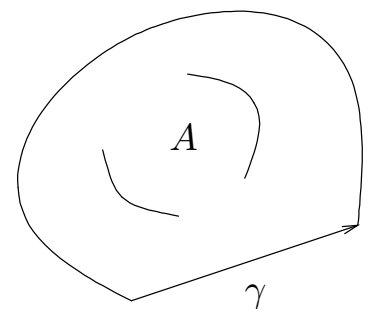

Figure 11: Example of globular cycle in higher dimension

Let $f$ be an $\omega$-functor from $\mathcal{C}$ to $\mathcal{D}$. Then $f$ induces for all $n \geq 0$ a linear morphism $f_{*}$ from $\mathbb{Z} \mathcal{C}_{n}$ to $\mathbb{Z} \mathcal{D}_{n}$ by setting $f_{*}(x)=f(x) \bmod \mathcal{D}_{\leqslant n-1}$ : this notation meaning that $f_{*}(x)=f(x)$ if $f(x)$ is $n$-dimensional and $f_{*}(x)=0$ otherwise. For $n \geq 2, f_{*}\left(s_{n-1}-\right.$ $\left.t_{n-1}\right)(x)=\left(s_{n-1}-t_{n-1}\right) f_{*}(x)$, therefore $f_{*} \partial^{g l}(x)=\partial^{g l} f_{*}(x)$. The latter equality is not anymore true if $x$ is 1-dimensional because an $\omega$-functor can contract 1-morphisms and because $\partial^{g l}(x)=\left(s_{0}(x), t_{0}(x)\right)$. So the globular homology does not yield a functor from $\omega C a t$ to $A b$ but only a functor from $\omega C a t_{1}$ to $A b$.

\subsection{Homological property}

Now we give a homological property of the globular complex to justify this construction. The starting point is the small category Glob defined as follows : the objects are all natural numbers and the arrows are generated by $s$ and $t$ in $G l o b(m, m-1)$ for any $m>0$ and quotiented by the relations $s s=s t, t s=t t$. We can depict Glob like this :

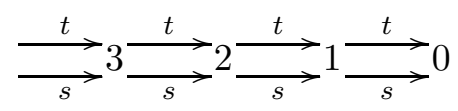

Definition 3.3. A globular set is a covariant functor from Glob to Sets. The corresponding category is denoted by $[G l o b, S e t s]$. A globular group is a covariant functor from Glob to the category Ab of abelian groups. The corresponding category is denoted by [Glob, Ab].

The notion of globular set already appears in many works and is certainly not new [Str98] Pen99] Bat98].

If $\mathcal{C}$ is an $\omega$-category, we denote by $\mathcal{C}_{n}$ the set of $n$-dimensional morphisms of $\mathcal{C}$ with $n \geqslant 0$.

Definition 3.4. Let $G l$ be the map from $\omega$-categories to globular groups defined as follows. If $n \geqslant 0$, set $G l(\mathcal{C})_{n}=\mathbb{Z C}_{n}$. For any $s, t \in G l o b(n+1, n)$, set $G l(s)(x)=s_{n}(x)$ and $G l(t)(x)=t_{n}(x)$. 
Unfortunately, $G l(-)$ is not a functor because an $\omega$-functor might be $n$-contracting for $n \geqslant 2$. That is, suppose that $f$ is an $\omega$-functor from $\mathcal{C}$ to $\mathcal{D}$ such that for a 2-morphism $x$ of $\mathcal{C}, f(x)$ is 1-dimensional. Then $G l(f)(x)=0 \in G l(\mathcal{D})_{2}$ and $s_{1} G l(f)(x)=0$ but $G l(f)\left(s_{1}(x)\right)=f\left(s_{1}(x)\right) \in G l(\mathcal{D})_{1}$ and $f\left(s_{1}(x)\right) \neq 0$.

If $M$ is a globular group, let $H(M)$ be the cokernel of the additive map from $M_{1}$ to $M_{0} \oplus M_{0}$ which maps $x$ to $(s(x), t(x))$. The map $H$ induces a right exact additive functor from $[G l o b, A b]$ to $A b$. Since $[G l o b, A b]$ has enough projectives, we can deal with the left derived functors $L_{n}(H)$ of $H$ (see Wei94 or Rot79 for the definition of projective object and right exact functor).

Proposition 3.1. For any $\omega$-category $\mathcal{C}$, we have $H_{*}^{g l}(\mathcal{C}) \cong L_{*}(H)(G l(\mathcal{C}))$.

Before proving this theorem, we need to recall standard facts about category of diagrams. We are going to solve exercice (2.3.13) of Wei94 because in the sequel we need a precise description of a family of projective globular groups which allows to resolve any globular group.

Let $e v_{k}$ be the functor from $[G l o b, A b]$ to $A b$ such that $e v_{k}(M)=M(k), k$ being a natural number. This functor is exact and by the special adjoint functor theorem has a left adjoint denoted by $k_{!}$. We need to explicit $k_{\text {! }}$ for the sequel.

Proposition 3.2. If $M$ is an abelian group, set

$$
k_{!}(M)(l)=\bigoplus_{h \in G l o b(k, l)} M_{h}
$$

where $M_{h}$ is a copy of $M$. If $x \in M$, let h.x be the corresponding element of $k_{!}(M)(l)$. If $f: l \longrightarrow l^{\prime}$ is an arrow of $I$, then we set $k_{!}(M)(f)(h . x)=(f h) . x$. Then $k_{!}$is a globular group and this is the left adjoint of $e v_{k}$.

Proof. Let $N$ be a globular group. We introduce the map

$$
F:[G l o b, A b]\left(k_{!}(M), N\right) \longrightarrow A b(M, N(k))
$$

defined by $F(u)(x)=u\left(I d_{k} \cdot x\right)$ and the map

$$
G: A b(M, N(k)) \longrightarrow[G l o b, A b]\left(k_{!}(M), N\right)
$$

defined by $G(v)(f . x)=N(f)(v(x))$. The arrow $G(v)$ is certainly a morphism of globular groups from $k_{!}(M)$ to $N$. In fact, if $l \stackrel{f}{\longrightarrow} l^{\prime}$ is an arrow of Glob and if $h . x$ is an element of $k_{!}(M)(l)$, then $G(v)(f h . x)=N(f h)(v(x))=N(f)(G(v)(h . x))$. Therefore the diagram

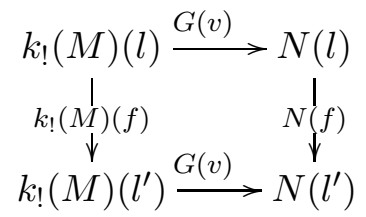


commutes. Now we have to verify that $F$ and $G$ are inverse of each other. Indeed,

$$
F(G(v))(x)=G(v)\left(I d_{k} \cdot x\right)=N(I d)(v(x))
$$

therefore $F(G(v))=v$. Conversely,

$$
G(F(u))(h . x)=N(h)(F(u)(x))=N(h)\left(u\left(I d_{k} \cdot x\right)\right)=u\left(k_{!}(M)(h)\left(I d_{k} \cdot x\right)\right)=u(h . x)
$$

therefore $G(F(u))=u$.

Now we set

$$
\mathcal{F}=\left\{\bigoplus_{k \in \mathbb{N}} k_{!}(L) / L \text { free module }\right\}
$$

And we can state the proposition

Proposition 3.3. All elements of $\mathcal{F}$ are projective globular groups. Any globular group can be resolved by elements of $\mathcal{F}$.

Proof. Let $X$ be a globular group. For any $k \in \mathbb{N}$, let $L_{k}$ be a free abelian group and $L_{k} \rightarrow X(k)$ an epimorphism of abelian groups. Then the epimorphisms $L_{k} \longrightarrow X(k)$ for all $k$ induce a natural transformation $\bigoplus_{k \in \mathbb{N}} k_{!}\left(L_{k}\right) \longrightarrow X$ which is certainly itself an epimorphism. Left adjoint functors and coproduct preserve projective objects Bor94. Hence the conclusion.

We are in position to prove the proposition :

Proposition 3.4. For any $\omega$-category $\mathcal{C}$, the equality $H_{*}^{g l}(\mathcal{C}) \cong L_{*}(H)(G l(\mathcal{C}))$ holds.

Proof. If $M$ is a globular group, we introduce the complex of abelian groups $\left(C_{*}^{g l}(M), \partial^{g l}\right)$ defined as follows : $C_{0}^{g l}(M)=M_{0} \oplus M_{0}$ and for $n \geqslant 1, C_{n}^{g l}(M)=M_{n}$, with the differential map $\partial^{g l}(x)=(s(x), t(x))$ if $x \in M_{1}$ and $\partial^{g l}(x)=s(x)-t(x)$ if $x \in M_{n}$ with $n \geqslant 2$. We have $H_{0}\left(C_{*}^{g l}(M), \partial\right)=H(M)$. Let $k$ be a natural number and let $L$ be a free abelian group. If $p>0$, let us prove that $H_{p}\left(C_{*}^{g l}\left(k_{!}(L)\right)\right)=0$. Let $X=x_{p}$ be a cycle of $C_{p}\left(k_{!}(L)\right)$. By construction, for all $p>k$, one has $k_{!}(L)(p)=0$. Therefore if $p>k$, then $X=0$ hence $H_{p}\left(C_{*}^{g l}\left(k_{!}(L)\right)\right)=0$ whenever $p>k$. Now let us see the case $p \leqslant k$. We have $0=$ $\partial^{g l}(X)=s_{p-1}\left(x_{p}\right)-t_{p-1}\left(x_{p}\right)$. Then there exists $x_{p}^{s}$ and $x_{p}^{t}$ such that $x_{p}=s^{k-p} \cdot x_{p}^{s}+t^{k-p} \cdot x_{p}^{t}$. The equality $s_{p-1}\left(x_{p}\right)=t_{p-1}\left(x_{p}\right)$ implies $s^{k-p+1} \cdot x_{p}^{s}+s^{k-p+1} \cdot x_{p}^{t}=t^{k-p+1} \cdot x_{p}^{s}+t^{k-p+1} \cdot x_{p}^{t}$. Therefore $x_{p}=0$.

Now we deduce from Proposition 3.3 that for any projective globular group $P$,

$$
H_{p}\left(C_{*}^{g l}(P), \partial\right)=0
$$


for all $p>0$. It is thus easy to check that for any natural number $p$, the equality $H_{p}\left(C_{*}^{g l}(M), \partial\right)=L_{p}(H)(M)$ holds. Indeed, the case $p=0$ is trivial and the commutative diagram

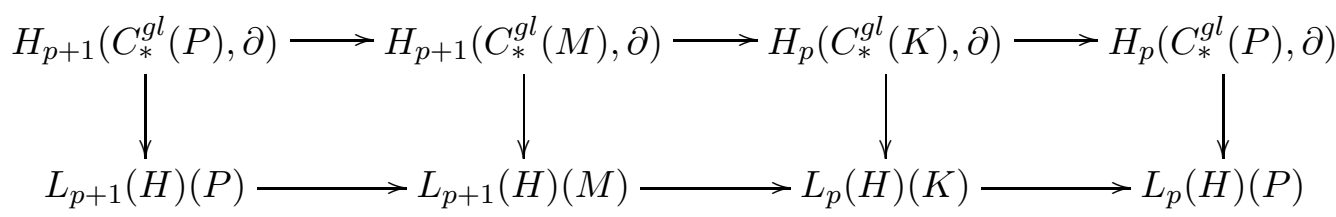

with $P$ projective allows to make the induction on $p$.

\section{Positive and negative corner homology of an w-category}

\subsection{The pasting scheme $\Lambda^{n}$ and the $\omega$-category $I^{n}$}

We need to describe precisely the $\omega$-category associated to the $n$-cube.

Definition 4.1. A pasting scheme is a triple $(A, E, B)$ where $A$ is a $\mathbb{N}$-graded set, and $E_{j}^{i}$ and $B_{j}^{i}$ two binary relations over $A_{i} \times A_{j}$ with $j \leqslant i$ satisfying

(1) the set $E_{i}^{i}$ is the diagonal of $A_{i}$

(2) for $k>0$, and for any $x \in A_{k}$, there exists $y \in A_{k-1}$ with $x E_{k-1}^{k} y$

(3) for $k<n, w E_{k}^{n} x$ if and only if there exists $u$ and $v$ such that $w E_{n-1}^{n} u E_{k}^{n-1} x$ and $w E_{n-1}^{n} v B_{k}^{n-1} x$

(4) if $w E_{n-1}^{n} z E_{k}^{n-1} x$, then either $w E_{k}^{n} x$ or there exists $v$ such that $w B_{n-1}^{n} v E_{k}^{n-1} x$

and such that $(A, B, E)$ satisfies the same properties. If $x \in A_{i}$, we set $\operatorname{dim}(x)=i$.

If $x$ is an element of the pasting scheme $(A, E, B)$, we denote by $R(x)$ the smallest pasting scheme of $(A, E, B)$ containing $x$.

Intuitively, a pasting scheme is a pasting of faces of several dimensions together Joh89. Kapranov and Voedvosky have their own formalization using some particular chain complexes of abelian groups [KV91]. We can see Figure 3 as a pasting scheme $(S, E, B)$. It suffices to set $S=\left\{\alpha, \beta, s_{1} A, t_{1} A, t_{1} B, A, B\right\}$ endowed with the binary relations $B_{1}^{2}=\left\{\left(A, s_{1} A\right),\left(B, s_{1} B\right)\right\}, E_{1}^{2}=\left\{\left(A, t_{1} A\right),\left(B, t_{1} B\right)\right\}, B_{0}^{2}=E_{0}^{2}=\{\}, B_{0}^{1}=$ $\left\{\left(s_{1} A, \alpha\right),\left(t_{1} A, \alpha\right),\left(t_{1} B, \alpha\right)\right\}, E_{0}^{1}=\left\{\left(s_{1} A, \beta\right),\left(t_{1} A, \beta\right),\left(t_{1} B, \beta\right)\right\}$. Figure 12 shows another more complicated example of pasting scheme. 


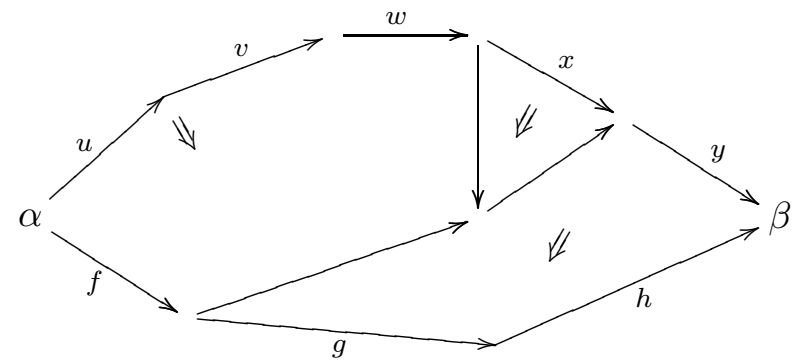

Figure 12: A pasting scheme

We only want here to recall the construction of the free $\omega$-category $I^{n}$ generated by the faces of the $n$-cube. For more details see [Cra95], for an analogous construction for simplices see [Str87], and for some explicit calculations on $I^{n}$ see [Ait86].

Set $\underline{n}=\{1, \ldots, n\}$ and let $\Lambda^{n}$ be the set of maps from $\underline{n}$ to $\{-, 0,+\}$. We say that an element $x$ of $\Lambda^{n}$ is of dimension $p$ if $x^{-1}(0)$ is a set of $p$ elements. We can identify the elements of $\Lambda^{n}$ with the words of length $n$ in the alphabet $\{-, 0,+\}$. The set $\Lambda^{n}$ is supposed to be graded by the dimension of its elements. The set $\Lambda^{0}$ is the set of maps from the empty set to $\{-, 0,+\}$ and therefore it is a singleton.

Let $y \in \Lambda^{i}$. Let $r_{y}$ be the map from $\left(\Lambda^{n}\right)_{i}$ to $\left(\Lambda^{n}\right)_{\operatorname{dim}(y)}$ defined as follows (with $\left.x \in\left(\Lambda^{n}\right)_{i}\right):$ for $k \in \underline{n}, x(k) \neq 0$ implies $r_{y}(x)(k)=x(k)$ and if $x(k)$ is the $l$-th zero of the sequence $x(1), \ldots, x(n)$, then $r_{y}(x)(k)=y(\ell)$. If for any $\ell$ between 1 and $i, y(\ell) \neq 0$ implies $y(\ell)=(-)^{\ell}$, then we set $b_{y}(x):=r_{y}(x)$. If for any $\ell$ between 1 and $i, y(\ell) \neq 0$ implies $y(\ell)=(-)^{\ell+1}$, then we set $e_{y}(x):=r_{y}(x)$. We thus introduce the following binary relations : the set $B_{j}^{i}$ of pairs $(x, z)$ in $\left(\Lambda^{n}\right)_{i} \times\left(\Lambda^{n}\right)_{j}$ such that there exists $y$ such that $z=b_{y}(x)$ and the set $E_{j}^{i}$ of pairs $(x, z)$ in $\left(\Lambda^{n}\right)_{i} \times\left(\Lambda^{n}\right)_{j}$ such that there exists $y$ such that $z=e_{y}(x)$. Then $\Lambda^{n}$ is a pasting scheme. We have

Theorem 4.1. If $X \subset \Lambda^{n}$, let $R(X)$ be the sub-pasting scheme of $\left(\Lambda^{n}, B_{j}^{i}, E_{j}^{i}\right)$ generated by $X$. There is one and only one $\omega$-category $I^{n}$ such that

1. the underlying set of $I^{n}$ is included in the set of sub-pasting schemes of $\left(\Lambda^{n}, B_{j}^{i}, E_{j}^{i}\right)$ and it contains all pasting schemes like $R(\{x\})$ where $x$ runs over $\Lambda^{n}$

2. all elements of $I^{n}$ are a composition of $R(\{x\})$ where $x$ runs over $\Lambda^{n}$

3. for $x$-dimensional with $p \geqslant 1$, one has

$$
\begin{aligned}
& s_{p-1}(R(\{x\}))=R\left(\left\{b_{y}(x), \operatorname{dim}(y)=p-1\right\}\right) \\
& t_{p-1}(R(\{x\}))=R\left(\left\{e_{y}(x), \operatorname{dim}(y)=p-1\right\}\right)
\end{aligned}
$$




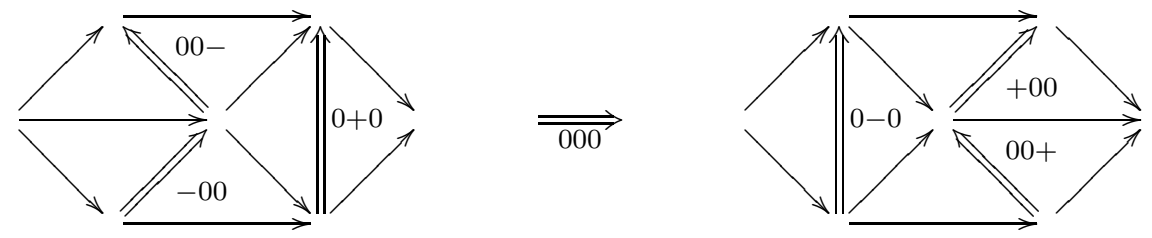

Figure 13: The $\omega$-category $I^{3}$

4. if $R(X)$ and $R(Y)$ are two elements of $I^{n}$ such that $t_{p}(R(X))=s_{p}(R(Y))$ for some $p$, then $R(X \cup Y) \in I^{n}$ and $R(X \cup Y)=R(X) *_{p} R(Y)$.

The oriented 2-cube is drawn in Figure 5 . With the rules exposed in the above theorem, we can calculate $s_{2} R(00)$. We have actually $s_{2} R(00)=R(\{-0,0+\})$. But $t_{0} R(-0)=$ $R(-+)=s_{0} R(0+)$. Then $s_{2} R(00)=R(\{-0\} \cup\{0+\})=R(-0) *_{0} R(0+)$.

The $\omega$-category generated by a 3 -cube is drawn in Figure 13. Let us give the example of the calculation of $s_{2} R(000)$. We have

$$
s_{2} R(000)=R(\{-00,0+0,00-\})=R(\{-00,0++\} \cup\{-0-, 0+0\} \cup\{00-, 0++\})
$$

since $0++,-0-, 0++\in R(\{-00,0+0,00-\})$. We verify easily that $t_{1} R(\{-00,0++\})=$ $s_{1} R(\{-0-, 0+0\})$ and $t_{1} R(\{-0-, 0+0\})=s_{1} R(\{00-, 0++\})$. Therefore

$$
s_{2} R(000)=R(\{-00,0++\}) *_{1} R(\{-0-, 0+0\}) *_{1} R(\{00-, 0++\}) .
$$

It is then easy to verify that $R(\{-00,0++\})=R(-00) *_{0} R(0++), R(\{-0-, 0+0\})=$ $R(-0-) *_{0} R(0+0)$ and $R(\{00-, 0++\})=R(00-) *_{0} R(0++)$.

The oriented 4-cube is represented in Figure 14.

\subsection{The cubical singular nerve}

The map which sends every $\omega$-category $\mathcal{C}$ to $\mathcal{N}^{\square}(\mathcal{C})_{*}=\omega \operatorname{Cat}\left(I^{*}, \mathcal{C}\right)$ induces a functor from $\omega C a t$ to the category of cubical sets. If $x$ is an element of $\omega \operatorname{Cat}\left(I^{n}, \mathcal{C}\right), \epsilon_{i}(x)$ is the $\omega$-functor from $I^{n+1}$ to $\mathcal{C}$ defined by $\epsilon_{i}(x)\left(k_{1} \ldots k_{n+1}\right)=x\left(k_{1} \ldots \widehat{k}_{i} \ldots k_{n+1}\right)$ for all $i$ between 1 and $n+1$ and $\partial_{i}^{\alpha}(x)$ is the $\omega$-functor from $I^{n-1}$ to $\mathcal{C}$ defined by $\partial_{i}^{\alpha}(x)\left(k_{1} \ldots k_{n-1}\right)=x\left(k_{1} \ldots k_{i-1} \alpha k_{i} \ldots k_{n-1}\right)$ for all $i$ between 1 and $n$.

The arrow $\partial_{i}^{\alpha}$ for a given $i$ such that $1 \leqslant i \leqslant n$ induces a natural transformation from $\omega \operatorname{Cat}\left(I^{n},-\right)$ to $\omega \operatorname{Cat}\left(I^{n-1},-\right)$ and therefore, by Yoneda, corresponds to an $\omega$-functor $\delta_{i}^{\alpha}$ from $I^{n-1}$ to $I^{n}$. This functor is defined on the faces of $I^{n-1}$ by $\delta_{i}^{\alpha}\left(k_{1} \ldots k_{n-1}\right)=$ $R\left(k_{1} \ldots[\alpha]_{i} \ldots k_{n-1}\right)$. The notation $[\ldots]_{i}$ means that the term inside the brackets are in the $i$-th place. 


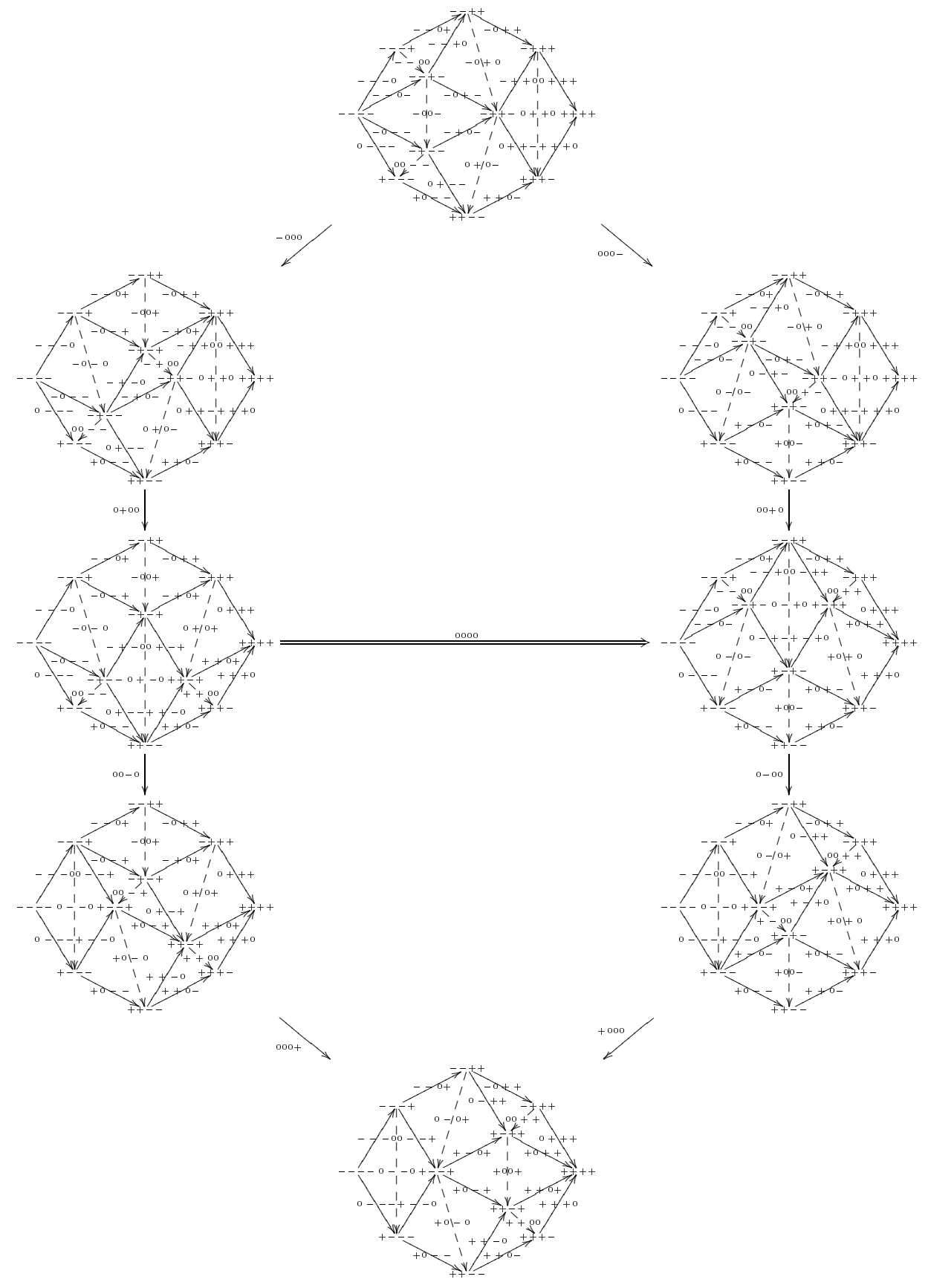

19

Figure 14: The $\omega$-category $I^{4}$ 
Definition 4.2. The cubical set $\omega \operatorname{Cat}\left(I^{*}, \mathcal{C}\right)$ is called the cubical singular nerve of the $\omega$ category $\mathcal{C}$.

This functor is a right adjoint. Its left adjoint is the functor $F$.

\subsection{Construction of the corner homologies}

The starting point is the cubical singular nerve $\omega \operatorname{Cat}\left(I^{*}, \mathcal{C}\right)$ of $\mathcal{C}$ which contains all $n$ cubes included in $\mathcal{C}$. The main idea to build the positive and negative corner homology of an $\omega$-category $\mathcal{C}$ is to separate the two differential maps $\partial^{-}=\sum_{i}(-1)^{i+1} \partial_{i}^{-}$and $\partial^{+}=$ $\sum_{i}(-1)^{i+1} \partial_{i}^{+}$and to separately consider the chain complexes of groups $\left(\mathbb{Z} \omega C a t\left(I^{*}, \mathcal{C}\right), \partial^{ \pm}\right)$ (a bit as in Gou95 where the author separates the horizontal and vertical differential maps of a bicomplex). However the following proposition holds :

Proposition 4.2. Both chain complexes of groups $\left(\mathbb{Z} \omega C \operatorname{Cat}\left(I^{*}, \mathcal{C}\right), \partial^{-}\right)$and $\left(\mathbb{Z} \omega \operatorname{Cat}\left(I^{*}, \mathcal{C}\right)\right.$, $\left.\partial^{+}\right)$are acyclic.

Proof. It turns out that $\epsilon_{1}$ is a chain retraction of $\left(\mathbb{Z} \omega C a t\left(I^{*}, \mathcal{C}\right), \partial^{-}\right)$. If $x \in \mathbb{Z} \omega C a t\left(I^{0}, \mathcal{C}\right)$, then $\partial^{\alpha} \epsilon_{1} x=\partial_{1}^{\alpha} \epsilon_{1} x=x$. And for $x \in \mathbb{Z} \omega C a t\left(I^{n}, \mathcal{C}\right)$, with $n \geqslant 1$, we have actually :

$$
\begin{aligned}
\partial^{\alpha} \circ \epsilon_{1}(x)+\epsilon_{1} \circ \partial^{\alpha}(x) & =\sum_{i=1}^{i=n+1}(-1)^{i+1} \partial_{i}^{\alpha} \epsilon_{1}(x)+\sum_{i=1}^{i=n}(-1)^{i+1} \epsilon_{1} \partial_{i}^{\alpha}(x) \\
& =x+\sum_{i=2}^{i=n+1}(-1)^{i+1} \epsilon_{1} \partial_{i-1}^{\alpha}(x)+\sum_{i=1}^{i=n}(-1)^{i+1} \epsilon_{1} \partial_{i}^{\alpha}(x) \\
& =x+\sum_{i=1}^{i=n}(-1)^{i} \epsilon_{1} \partial_{i}^{\alpha}(x)+\sum_{i=1}^{i=n}(-1)^{i+1} \epsilon_{1} \partial_{i}^{\alpha}(x) \\
& =x
\end{aligned}
$$

The previous proposition entails the following definition :

Definition 4.3. Let $\mathcal{C}$ be an $\omega$-category and $\alpha \in\{-,+\}$. We denote by $\omega C$ at $\left(I^{n}, \mathcal{C}\right)^{\alpha}$ the subset of elements $x$ of $\omega$ Cat $\left(I^{n}, \mathcal{C}\right)$ satisfying the following conditions :

- the element $x$ is a non degenerate element of the cubical nerve

- any element of the form $\partial_{i_{1}}^{\alpha} \ldots \partial_{i_{p}}^{\alpha}(x)$ is non degenerate in the cubical nerve. 
Then $\partial^{\alpha}\left(\mathbb{Z} \omega C a t\left(I^{*+1}, \mathcal{C}\right)^{\alpha}\right) \subset \mathbb{Z} \omega C a t\left(I^{*}, \mathcal{C}\right)^{\alpha}$ by construction. We thus set

$$
H_{*}^{\alpha}(\mathcal{C}, \mathbb{Z})=H_{*}\left(\mathbb{Z} \omega \operatorname{Cat}\left(I^{*}, \mathcal{C}\right)^{\alpha}, \partial^{\alpha}\right)
$$

and we call these homology theories the negative (or positive according to $\alpha$ ) corner homology of $\mathcal{C}$. The cycles are called the negative (or positive) corners of $\mathcal{C}$. The maps $H_{*}^{ \pm}$induce functors from $\omega \mathrm{Cat}_{1}$ to $\mathrm{Ab}$.

The second part of the definition is essential. Indeed if $a$ is a 1-dimensional morphism of $\mathcal{C}$, then the following element of $\omega \operatorname{Cat}\left(I^{2}, \mathcal{C}\right)$ is non degenerate although its image by $\partial_{1}^{-}$ and $\partial_{2}^{-}$are degenerate elements of $\operatorname{Cat}\left(I^{1}, \mathcal{C}\right)$ :

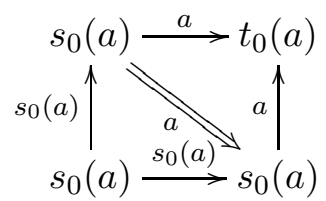

The following proposition characterizes the elements of $\omega \operatorname{Cat}\left(I^{*}, \mathcal{C}\right)^{\alpha}$

Proposition 4.3. Assume that $x$ is an element of $\omega \operatorname{Cat}\left(I^{*}, \mathcal{C}\right)$. Then $x$ is in $\omega \operatorname{Cat}\left(I^{*}, \mathcal{C}\right)^{\alpha}$ if and only if all $x(\alpha \ldots . \ldots \alpha)$ (the notation $\alpha \ldots 0 \ldots \alpha$ meaning that 0 appears only once) are 1-dimensional morphisms of $\mathcal{C}$.

Proof. If $x$ is in $\omega \operatorname{Cat}\left(I^{n}, \mathcal{C}\right)^{\alpha}$, then all $\partial_{i_{1}}^{\alpha} \ldots \partial_{i_{p}}^{\alpha}(x)$ are non degenerate in the cubical nerve. But $y \in \omega \operatorname{Cat}\left(I^{1}, \mathcal{C}\right)$ is non degenerate if and only if $y(R(0))$ is 1-dimensional. Hence the necessity of the condition. Conversely assume that $x \notin \omega \operatorname{Cat}\left(I^{n}, \mathcal{C}\right)^{\alpha}$. Then there exists $i$ between 1 and $n$ such that $\partial_{i_{1}}^{\alpha} \ldots \partial_{i_{p}}^{\alpha} x=\epsilon_{i}(z)$ with $p<n$ and some $i_{1}, \ldots, i_{p}$ and with $z \in \omega \operatorname{Cat}\left(I^{n-p-1}, \mathcal{C}\right)$. Then

$$
\left\{x\left(\alpha \ldots[-]_{i} \ldots \alpha\right), x\left(\alpha \ldots[0]_{i} \ldots \alpha\right), x\left(\alpha \ldots[+]_{i} \ldots \alpha\right)\right\}
$$

is a singleton for some $i$ therefore $x\left(\alpha \ldots[0]_{i} \ldots \alpha\right)$ is 0 -dimensional. Hence the sufficiency of the condition.

As the globular homology, the corner homologies do not yield functors from $\omega$ Cat to $A b$.

\subsection{Examples of corners}

Proposition 4.4. Let $\mathcal{C}$ be an $\omega$-category. The group $H_{0}^{-}(\mathcal{C})$ is the free abelian group generated by the final states of $\mathcal{C}$. The group $H_{0}^{+}(\mathcal{C})$ is the free abelian group generated by the initial states of $\mathcal{C}$. 


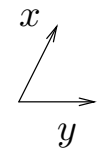

Figure 15: A 1-dimensional negative corner

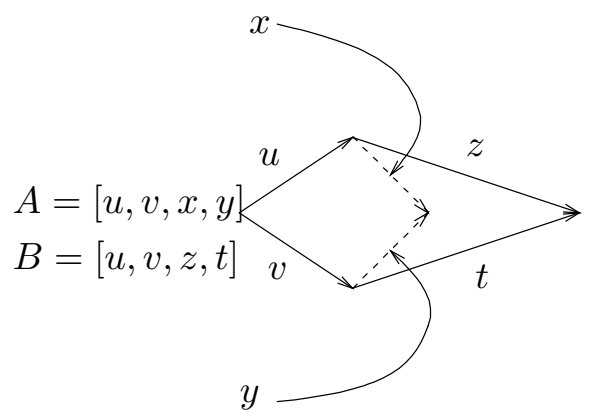

Figure 16: A 2-dimensional negative corner

Proof. Obvious.

There is in Figure 15 a very simple example of 1-dimensional negative corner. It consists of two $\omega$-functors $x$ and $y$ from $I^{1}$ to $\mathcal{C}$ such that $x(R(-))=y(R(-))$ and such that $x(R(0))$ and $y(R(0))$ are 1-dimensional. Figure 16 shows an example of 2-dimensional negative corner. If we suppose $A$ and $B$ to be oriented such that $s_{1} A=u *_{0} x, t_{1} A=v *_{0} y$, $s_{1} B=u *_{0} z$ and $t_{1} B=v *_{0} t$, then $A-B$ is a negative corner.

\section{Filling of shells in the cubical singular nerve}

Now here is a technical tool which will enable us to construct some operations on the cubical singular nerve of a globular $\omega$-category (Section 6 and Section 10) and to construct the two Hurewicz morphisms (Section 7). The notion of shell and of filling of (thin or not) shells already appears in BH81b in the framework of $\omega$-groupoids, in [AA89] in the framework of cubical $\omega$-categories (see Definition 10.1).

\subsection{Recall about the freeness of $I^{n}$}

A key property of $I^{n}$ is its freeness. It means the following fact. Let $p$ be some natural number. Let us call a realization $\left(\Lambda^{p}, f_{i}\right)$ of $\left(\Lambda^{p}, E, B\right)$ in an $\omega$-category $\mathcal{C}$ a family of maps $f_{i}$ from $\left(\Lambda^{p}\right)_{i}$ to $\tau_{i} \mathcal{C}$, where $\tau_{i} \mathcal{C}$ is the $\omega$-category obtained by only keeping the cells of $\mathcal{C}$ 
of dimension lower or equal than $i$. The realization $\left(\Lambda^{p}, f_{i}\right)$ is called $n$-extendable if there exists only one functor $f$ from $\tau_{n} I^{p}$ to $\mathcal{C}$ such that for any $k \leqslant n$, the commutative diagram holds

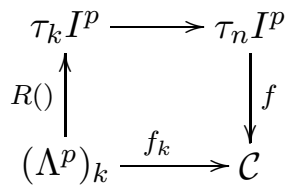

By convention, any realization $\left(\Lambda^{p}, f_{i}\right)$ is 0 -appropriate and is already 0-extendable since $f_{0}$ is already a functor from $\left(I^{p}\right)_{0}$ to $\mathcal{C}$. Suppose set up a notion of $n$-appropriate realization. Then we have

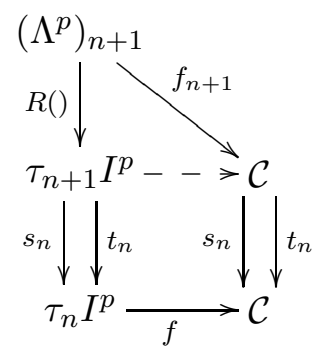

A realization $\left(\Lambda^{p}, f_{i}\right)$ is $(n+1)$-appropriate if $s_{n} f_{n+1}=f s_{n} R$ and $t_{n} f_{n+1}=f t_{n} R$, and in this case, $\left(\Lambda^{p}, f_{i}\right)$ is $(n+1)$-extendable (cf [Joh89] page 224). Thus to construct an $\omega$-functor from $I^{n}$ for some natural number $n$ to an $\omega$-category $\mathcal{C}$, it suffices to construct a realization which is appropriate in all dimensions.

\subsection{Filling of shells using the freeness of $I^{n}$}

Definition 5.1. An element $x \in \omega C$ at $\left(I^{n}, \mathcal{C}\right)$ is thin if $x\left(R\left(0_{n}\right)\right)$ is of dimension strictly lower than n. An element which is not thin is called thick.

Definition 5.2. A n-shell in the cubical singular nerve is a family of $2(n+1)$ elements $x_{i}^{ \pm}$ of $\omega \operatorname{Cat}\left(I^{n}, \mathcal{C}\right)$ such that $\partial_{i}^{\alpha} x_{j}^{\beta}=\partial_{j-1}^{\beta} x_{i}^{\alpha}$ for $1 \leqslant i<j \leqslant n+1$ and $\alpha, \beta \in\{-,+\}$.

Definition 5.3. The $n$-shell $\left(x_{i}^{ \pm}\right)$is fillable if

1. the sets $\left\{x_{i}^{(-)^{i}}, 1 \leqslant i \leqslant n+1\right\}$ and $\left\{x_{i}^{(-)^{i+1}}, 1 \leqslant i \leqslant n+1\right\}$ have each one exactly one thick element and if the other ones are thin.

2. if $x_{i_{0}}^{(-)^{i_{0}}}$ and $x_{i_{1}}^{(-)^{i_{1}+1}}$ are these two thick elements then there exists $u \in \mathcal{C}$ such that $s_{n}(u)=x_{i_{0}}^{(-)^{i_{0}}}\left(0_{n}\right)$ and $t_{n}(u)=x_{i_{1}}^{(-)^{i_{1}+1}}\left(0_{n}\right)$. 
The main proposition of this section is the following one, which is an analogue of AA89 Proposition 2.7.3.

Proposition 5.1. Let $\left(x_{i}^{ \pm}\right)$be a fillable $n$-shell with $u$ as above. Then there exists one and only one element $x$ of $\omega C$ at $\left(I^{n+1}, \mathcal{C}\right)$ such that $x\left(0_{n+1}\right)=u$, and for $1 \leqslant i \leqslant n+1$, and $\alpha \in\{-,+\}$ such that $\partial_{i}^{\alpha} x=x_{i}^{\alpha}$.

Proof. The underlying idea of the proof is as follows. If one wants to define an $\omega$-functor from $I^{n+1}$ to an $\omega$-category $\mathcal{C}$, it suffices to construct $2(n+1) \omega$-functors from the $2(n+1)$ $n$-faces of $I^{n+1}$ to $\mathcal{C}$ which coincide on the intersection of their definition domains and to fill correctly the interior of $I^{n+1}$.

We have necessarily $x\left(k_{1} \ldots[ \pm]_{i} \ldots k_{n}\right)=x_{i}^{ \pm}\left(k_{1} \ldots k_{n}\right)$ and $x\left(0_{n+1}\right)=u$. Therefore there is at most one such realization $\left(\Lambda^{n+1}, x_{i}\right)$. It suffices to show that $x$ is $(n+1)$ extendable. It is certainly 0-appropriate therefore 0-extendable. Suppose we have proved that this realization is $p$-appropriate and therefore $p$-extendable for every $p<n+1$. First suppose that $p<n$. We want to prove that $\left(\Lambda^{n+1}, x_{i}\right)$ is $(p+1)$-appropriate, i.e. that $s_{p} x\left(k_{1} \ldots k_{n+1}\right)=x s_{p} R\left(k_{1} \ldots k_{n+1}\right)$ and $t_{p} x\left(k_{1} \ldots k_{n+1}\right)=x t_{p} R\left(k_{1} \ldots k_{n+1}\right)$ for $k_{1} \ldots k_{n+1}$ of dimension $p+1$.

Let us verify the first equality. We have

$$
\begin{aligned}
s_{p} x\left(k_{1} \ldots k_{n+1}\right) & =s_{p}\left(\partial_{i}^{k_{i}} x\right)\left(k_{1} \ldots \widehat{k_{i}} \ldots k_{n+1}\right) \text { since } p+1<n+1 \\
& =s_{p} x_{i}^{k_{i}}\left(k_{1} \ldots \widehat{k_{i}} \ldots k_{n+1}\right) \\
& =x_{i}^{k_{i}} s_{p} R\left(k_{1} \ldots \widehat{k_{i}} \ldots k_{n+1}\right) \text { since the } x_{i}^{\alpha} \text { are } \omega \text {-functors }
\end{aligned}
$$

But $s_{p} R\left(k_{1} \ldots \widehat{k_{i}} \ldots k_{n+1}\right)=\Psi\left(R\left(X_{1}\right), \ldots, R\left(X_{s}\right)\right)$ where $\Psi$ consists only of compositions of $X_{h}$ of dimension lower than $p$. Then

$$
\begin{aligned}
s_{p} x\left(k_{1} \ldots k_{n+1}\right) & =\Psi\left(x_{i}^{k_{i}} R\left(X_{1}\right), \ldots, x_{i}^{k_{i}} R\left(X_{s}\right)\right) \\
& =\Psi\left(\left(\partial_{i}^{k_{i}} x\right) R\left(X_{1}\right), \ldots,\left(\partial_{i}^{k_{i}} x\right) R\left(X_{s}\right)\right) \\
& =x \Psi\left(\delta_{i}^{k_{i}} R\left(X_{1}\right), \ldots, \delta_{i}^{k_{i}} R\left(X_{s}\right)\right) \text { since } x \text { is } p \text {-extendable } \\
& =x s_{p} R\left(k_{1} \ldots k_{n+1}\right) \text { since } \delta_{i}^{k_{i}} \text { is an } \omega \text {-functor }
\end{aligned}
$$

It remains to prove that $x$ is $(n+1)$-extendable. We have to prove that $s_{n} x\left(0_{n+1}\right)=$ $x s_{n} R\left(0_{n+1}\right)$ and $t_{n} x\left(0_{n+1}\right)=x t_{n} R\left(0_{n+1}\right)$. Let us verify the first equality. We have $s_{n} R\left(0_{n+1}\right)=\Psi^{\prime}\left(\delta_{i_{0}}^{(-)^{i_{0}}}\left(0_{n}\right), Y_{1}, \ldots, Y_{s}\right)$ where $\Psi^{\prime}$ is a function containing only composition maps and where $Y_{1}, \ldots, Y_{s}$ are elements of $I^{n+1}$ of the form $R(\{x\})$ where $x$ is a face of the $(n+1)$-cube. Then

$$
x s_{n} R\left(0_{n+1}\right)=x \Psi^{\prime}\left(\delta_{i_{0}}^{(-)^{i} 0}\left(0_{n}\right), Y_{1}, \ldots, Y_{s}\right)
$$




$$
\begin{aligned}
& =\Psi^{\prime}\left(x\left(\delta_{i_{0}}^{(-)^{i_{0}}}\left(0_{n}\right)\right), x\left(Y_{1}\right), \ldots, x\left(Y_{s}\right)\right) \text { since } x \text { is } n \text {-extendable } \\
& =x\left(\delta_{i_{0}}^{(-)^{i_{0}}}\left(0_{n}\right)\right) \text { since } \operatorname{dim}\left(x\left(Y_{1}\right)\right), \ldots, \operatorname{dim}\left(x\left(Y_{s}\right)\right)<n \\
& =x_{i_{0}}^{(-)^{i_{0}}}\left(0_{n}\right) \\
& =s_{n}(u) \text { by definition of } u \\
& =s_{n} x\left(0_{n+1}\right) \text { by definition of } x
\end{aligned}
$$

\section{Two new simplicial nerves}

As an immediate application of Section 5 , we construct two families of connections on the cubical singular nerve of any $\omega$-category. They will be useful in the sequel.

\subsection{Cubical set with connections}

Definition 6.1. AA89 A cubical set with connections consists of a cubical set

$$
\left(\left(K_{n}\right)_{n \geqslant 0}, \partial_{i}^{\alpha}, \epsilon_{i}\right)
$$

together with two additional families of degeneracy maps

$$
\Gamma_{i}^{\alpha}: K_{n} \longrightarrow K_{n+1}
$$

with $\alpha \in\{-,+\}, n \geqslant 1$ and $1 \leqslant i \leqslant n$ and satisfying the following axioms :

1. $\partial_{i}^{\alpha} \Gamma_{j}^{\beta}=\Gamma_{j-1}^{\beta} \partial_{i}^{\alpha}$ for all $i<j$ and all $\alpha, \beta \in\{-,+\}$

2. $\partial_{i}^{\alpha} \Gamma_{j}^{\beta}=\Gamma_{j}^{\beta} \partial_{i-1}^{\alpha}$ for all $i>j+1$ and all $\alpha, \beta \in\{-,+\}$

3. $\partial_{j}^{ \pm} \Gamma_{j}^{ \pm}=\partial_{j+1}^{ \pm} \Gamma_{j}^{ \pm}=I d$

4. $\partial_{j}^{ \pm} \Gamma_{j}^{\mp}=\partial_{j+1}^{ \pm} \Gamma_{j}^{\mp}=\epsilon_{j} \partial_{j}^{ \pm}$

5. $\Gamma_{i}^{ \pm} \Gamma_{j}^{ \pm}=\Gamma_{j+1}^{ \pm} \Gamma_{i}^{ \pm}$if $i \leqslant j$

6. $\Gamma_{i}^{ \pm} \Gamma_{j}^{\mp}=\Gamma_{j+1}^{\mp} \Gamma_{i}^{ \pm}$if $i<j$

7. $\Gamma_{i}^{ \pm} \Gamma_{j}^{\mp}=\Gamma_{j}^{\mp} \Gamma_{i-1}^{ \pm}$if $i>j+1$

8. $\Gamma_{i}^{ \pm} \epsilon_{j}=\epsilon_{j+1} \Gamma_{i}^{ \pm}$if $i<j$

9. $\Gamma_{i}^{ \pm} \epsilon_{j}=\epsilon_{i} \epsilon_{i}$ if $i=j$ 
10. $\Gamma_{i}^{ \pm} \epsilon_{j}=\epsilon_{j} \Gamma_{i-1}^{ \pm}$if $i>j$

There is an obviously defined small category $\Gamma$, such that the category of cubical sets with connections is exactly the category of presheaves over $\Gamma$. Hence the category of cubical set with connections is denoted by Set ${ }^{\Gamma^{o p}}$.

The category of cubical sets with connections equipped with the non 1-contracting morphisms is denoted by Set $_{1}^{\Gamma^{o p}}$.

Looking back to the cubical singular nerve of a topological space, we can endow it with connections as follows :

$$
\begin{aligned}
& \Gamma_{i}^{-}(f)\left(x_{1}, \ldots, x_{p}\right)=f\left(x_{1}, \ldots, \max \left(x_{i}, x_{i+1}\right), \ldots, x_{p}\right) \\
& \Gamma_{i}^{+}(f)\left(x_{1}, \ldots, x_{p}\right)=f\left(x_{1}, \ldots, \min \left(x_{i}, x_{i+1}\right), \ldots, x_{p}\right)
\end{aligned}
$$

By keeping in a cubical set with connections only the morphisms $\Gamma_{i}^{-}$, or by exchanging the role of the face maps $\partial_{i}^{+}$and $\partial_{i}^{-}$and by keeping only the morphisms $\Gamma_{i}^{+}$, we obtain exactly a cubical set with connections in the sense of Brown-Higgins.

\subsection{Construction of connections on the cubical singular nerve}

Theorem 6.1. Let $\mathcal{C}$ be an $\omega$-category and let $n$ be a natural number greater or equal than 1. For any $x$ in $\omega \operatorname{Cat}\left(I^{n}, \mathcal{C}\right)$ and for any $i$ between 1 and $n$, we introduce two realizations $\Gamma_{i}^{-}(x)$ and $\Gamma_{i}^{+}(x)$ from $\Lambda^{n+1}$ to $\mathcal{C}$ by setting

$$
\begin{aligned}
\Gamma_{i}^{-}(x)\left(k_{1} \ldots k_{n+1}\right) & =x\left(k_{1} \ldots \max \left(k_{i}, k_{i+1}\right) \ldots k_{n+1}\right) \\
\Gamma_{i}^{+}(x)\left(k_{1} \ldots k_{n+1}\right) & =x\left(k_{1} \ldots \min \left(k_{i}, k_{i+1}\right) \ldots k_{n+1}\right)
\end{aligned}
$$

where the set $\{-, 0,+\}$ is ordered by $-<0<+$. Then $\Gamma_{i}^{-}(x)$ and $\Gamma_{i}^{+}(x)$ yield $\omega$-functors from $I^{n+1}$ to $\mathcal{C}$, meaning two elements of $\omega$ Cat $\left(I^{n+1}, \mathcal{C}\right)$. Moreover, in this way, the cubical nerve of $\mathcal{C}$ is equipped with a structure of cubical complex with connections.

Proof. The construction of $\Gamma_{i}^{ \pm}$is exactly the same as the one of connections on the cubical singular nerve of a topological space. Thus there is nothing to verify in the axioms of cubical complex with connections except the relations mixing the two families of degeneracies $\Gamma_{i}^{+}$ and $\Gamma_{i}^{-}$: all other axioms are already verified in BH81b. Therefore it remains to verify that $\Gamma_{i}^{ \pm} \Gamma_{j}^{\mp}=\Gamma_{j+1}^{\mp} \Gamma_{i}^{ \pm}$if $i<j$, and $\Gamma_{i}^{ \pm} \Gamma_{j}^{\mp}=\Gamma_{j}^{\mp} \Gamma_{i-1}^{ \pm}$if $i>j+1$, that can be done quickly. The only remaining point to be verified is that all realizations $\Gamma_{i}^{ \pm}(x)$ yield $\omega$-functors.

If $x$ is an element of $\omega C a t\left(I^{1}, \mathcal{C}\right)$, then we can depict $\Gamma_{1}^{-}(x)$ as in Figure 17 and $\Gamma_{1}^{+}(x)$ as in Figure 18. We see immediately that $\Gamma_{1}^{-}(x)$ and $\Gamma_{1}^{+}(x)$ yield two elements of $\omega C a t\left(I^{2}, \mathcal{C}\right)$.

Suppose we have proved that all realizations $\Gamma_{i}^{ \pm}(x)$ for $1 \leqslant i \leqslant n$ yield $\omega$-functors if $x \in \omega \operatorname{Cat}\left(I^{n}, \mathcal{C}\right)$. Now we want to prove that all realizations $\Gamma_{i}^{ \pm}(y)$ for $1 \leqslant i \leqslant n+1$ yield $\omega$-functors if $y \in \omega \operatorname{Cat}\left(I^{n+1}, \mathcal{C}\right)$. 


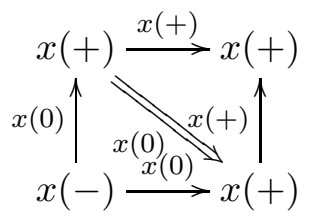

Figure 17: $\Gamma_{1}^{-}(x)$

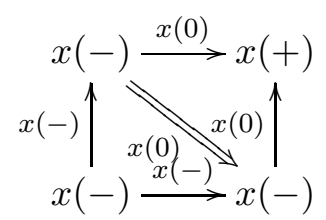

Figure 18: $\Gamma_{1}^{+}(x)$

Because of the axioms of cubical set with connections and because of the induction hypothesis, the $\left(\partial_{j}^{\alpha} \Gamma_{i}^{ \pm}(y)\right)$ are $\omega$-functor from $I^{n}$ to $\mathcal{C}$. The family $\left(\partial_{j}^{\alpha} \Gamma_{i}^{ \pm}(y)\right)$ is also a $n$-shell. We can fill it in a canonical way because the top dimensional elements are the same.

We denote by $\operatorname{Sets}^{\Delta^{o p}}$ the category of simplicial sets [May67. If $A$ is a simplicial set, the axioms of simplicial sets imply that $C(A)=\left(\mathbb{Z} A_{*}, \partial=\sum(-1)^{i} \partial_{i}\right)$, where $\mathbb{Z} A_{n}$ means the free abelian group generated by the set $A_{n}$, is a chain complex. It is called the unnormalized chain complex of $A$. The normalized chain complex of $A$ is the quotient chain complex $N(A)=C(A) / D(A)$ where $D(A)$ is the sub-complex of $C(A)$ generated by the degenerate elements. It turns out that the canonical morphism of chain complex from $C(A)$ to $N(A)$ is a quasi-isomorphism Wei94.

As a consequence of the previous construction, we obtain two new simplicial nerves.

Proposition 6.2. Let $\mathcal{C}$ be an $\omega$-category and $\alpha \in\{-,+\}$. We set

$$
\mathcal{N}_{n}^{\alpha}(\mathcal{C})=\omega \operatorname{Cat}\left(I^{n+1}, \mathcal{C}\right)^{\alpha}
$$

and for all $n \geqslant 0$ and all $0 \leqslant i \leqslant n$,

$$
\partial_{i}: \mathcal{N}_{n}^{\alpha}(\mathcal{C}) \longrightarrow \mathcal{N}_{n-1}^{\alpha}(\mathcal{C})
$$

is the arrow $\partial_{i+1}^{\alpha}$, and

$$
\epsilon_{i}: \mathcal{N}_{n}^{\alpha}(\mathcal{C}) \longrightarrow \mathcal{N}_{n+1}^{\alpha}(\mathcal{C})
$$


is the arrow $\Gamma_{i+1}^{\alpha}$. We obtain this way a simplicial set

$$
\left(\mathcal{N}_{*}^{\alpha}(\mathcal{C}), \partial_{i}, \epsilon_{i}\right)
$$

called the negative (or positive according to $\alpha$ ) corner simplicial nerve of $\mathcal{C}$. The non normalized chain complex associated to it gives exactly the corner homology of $\mathcal{C}$ (in degree greater than or equal to 1). The maps $\mathcal{N}^{\alpha}$ induces a functor from $\omega C_{\text {Cat }}$ to Sets $^{\Delta^{o p}}$.

Proof. The axioms of simplicial sets are immediate consequences of the axioms of cubical set with connections.

Notice that the indices are shifted by one. Intuitively, these simplicial nerves consist of cutting an oriented $n$-hypercube by an hyperplane close to a corner (the negative one or the positive one) : the intersection we get is the oriented $(n-1)$-simplex in sense of [Str87.

\section{The oriented Hurewicz morphisms}

In this section, we construct natural morphisms from the globular homology of a $\omega$-category $\mathcal{C}$ to its two corner homology theories. We call these maps the negative and positive oriented Hurewicz morphisms. Intuitively, they map any oriented loop with corners to its corresponding negative or positive corners (except for the 0 -dimensional, see below).

\subsection{The 0-dimensional case}

The projection from $\mathbb{Z} \mathcal{C}_{0} \times \mathbb{Z} \mathcal{C}_{0}$ to $\mathbb{Z} \mathcal{C}_{0}$ on the first (resp. the second) component yields a natural group morphism from $H_{0}^{g l}(\mathcal{C})$ to $H_{0}^{-}(\mathcal{C})\left(\right.$ resp. $H_{0}^{+}(\mathcal{C})$ ). Indeed if

$$
X=\left(s_{0}(x-y), t_{0}(x-y)\right),
$$

then the first component (resp. the second one) of $X$ induces 0 on the corner homology. We obtain thus a natural morphism $h_{0}^{ \pm}$from $H_{0}^{g l}(\mathcal{C})$ to $H_{0}^{ \pm}(\mathcal{C})$.

\subsection{The 1-dimensional case}

If $x$ is a 1-dimensional morphism of $\mathcal{C}$, let $\square_{1}(x)$ be the element of $\omega \operatorname{Cat}\left(I^{1}, \mathcal{C}\right)$ defined by

$$
\square_{1}(x)(R(-))=s_{0}(x), \square_{1}(x)(R(0))=x, \square_{1}(x)(R(+))=t_{0}(x) .
$$

We extend $\square_{1}$ by linearity. If $z$ is a 2-dimensional morphism of $\mathcal{C}$, let $\square_{2}^{-}(z)$ be the element of $\omega \operatorname{Cat}\left(I^{2}, \mathcal{C}\right)$ defined by the diagram

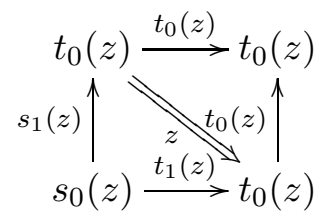


and let $\square_{2}^{+}(z)$ be the element of $\omega \operatorname{Cat}\left(I^{2}, \mathcal{C}\right)$ defined by

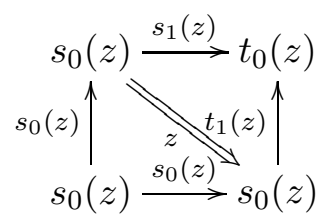

We extend $\square_{2}^{-}()$and $\square_{2}^{+}()$by linearity. We get thus the following proposition

Proposition 7.1. The natural linear map $h_{1}^{ \pm}$from $\mathbb{Z} \mathcal{C}_{1}$ to $\mathbb{Z} \omega C$ Cat $\left(I^{1}, \mathcal{C}\right)^{ \pm}$which maps $x_{1}$ to $\square_{1}\left(x_{1}\right)$ induces a natural map (still denoted by $h_{1}^{ \pm}$) from $H_{1}^{g l}(\mathcal{C})$ to $H_{1}^{ \pm}(\mathcal{C})$. We call it the 1-dimensional oriented Hurewicz morphism.

Proof. The proof is quite simple. If $x_{1}$ is a 1-dimensional globular cycle, then

$$
\partial^{ \pm} \square_{1}\left(x_{1}\right)=\square_{1}\left(x_{1}\right)( \pm)=0
$$

because of the definition of $\square_{1}\left(x_{1}\right)$. And a 1-dimensional globular boundary $s_{1}\left(x_{2}\right)-t_{1}\left(x_{2}\right)$ is mapped to $\partial^{ \pm}\left(\square_{2}^{-}\left(x_{2}\right)\right)$.

\subsection{The higher dimensional case}

Proposition 7.2. For any natural number $n$ greater or equal than 2, there exists a unique natural map $\square_{n}^{-}$from $\mathcal{C}_{n}$ (the $n$-dimensional cells of $\mathcal{C}$ ) to $\omega C$ at $\left(I^{n}, \mathcal{C}\right)$ such that

1. the equality $\square_{n}^{-}(x)\left(0_{n}\right)=x$ holds.

2. if $n \geqslant 3$ and $1 \leqslant i \leqslant n-2$, then $\partial_{i}^{ \pm} \square_{n}^{-}=\Gamma_{n-2}^{-} \partial_{i}^{ \pm} \square_{n-1}^{-} s_{n-1}$.

3. if $n \geqslant 2$ and $n-1 \leqslant i \leqslant n$, then $\partial_{i}^{-} \square_{n}^{-}=\square_{n-1}^{-} d_{n-1}^{(-)^{i}}$ and $\partial_{i}^{+} \square_{n}^{-}=\epsilon_{n-1} \partial_{n-1}^{+} \square_{n-1}^{-} s_{n-1}$. Moreover for $1 \leqslant i \leqslant n$, we have $\partial_{i}^{ \pm} \square_{n}^{-} s_{n} u=\partial_{i}^{ \pm} \square_{n}^{-} t_{n} u$ for any $(n+1)$-morphism $u$.

Proof. Let $k_{1} \ldots k_{n-1} \in \Lambda^{n-1}$. Then the natural map $e v_{k_{1} \ldots k_{n-1}} \partial_{i}^{ \pm} \square_{n}^{-}$from $\mathcal{C}_{n}$ to $\mathcal{C}_{n-1}$ which sends $x \in \mathcal{C}_{n}$ to $\left(\partial_{i}^{ \pm} \square_{n}^{-} x\right)\left(k_{1} \ldots k_{n-1}\right)$ corresponds by Yoneda to an $\omega$-functor $f_{k_{1} \ldots k_{n-1}}$ from $2_{n-1}$ to $2_{n}$, where $2_{n-1}$ (resp. $2_{n}$ ) is the free $\omega$-category generated by a $(n-1)$-morphism $A$ (resp. a $n$-morphism $B$ ). Set

$$
f_{k_{1} \ldots k_{n-1}}(A)=d_{n_{k_{1} \ldots k_{n-1}}}^{\alpha_{k_{1} \ldots k_{n-1}}}(B)
$$

with still the convention $d^{-}=s$ and $d^{+}=t$. Then $f_{k_{1} \ldots k_{n-1}}=d_{n_{k_{1} \ldots k_{n-1}}}^{\alpha_{k_{1} \ldots k_{n-1}}}$ because of the freeness of $2_{n-1}$. Moreover, the inequality $n_{k_{1} \ldots k_{n-1}} \leqslant n-1$ holds. Therefore

$$
\begin{aligned}
\left(\partial_{i}^{ \pm} \square_{n}^{-} s_{n} u\right)\left(k_{1} \ldots k_{n-1}\right) & =d_{n_{k_{1} \ldots k_{n-1}}}^{\alpha_{k_{1} \ldots k_{n-1}}} s_{n} u \\
& =d_{n_{k_{1} \ldots k_{n-1}}}^{\alpha_{k_{1} \ldots k_{n-1}}} t_{n} u \\
& =\left(\partial_{i}^{ \pm} \square_{n}^{-} t_{n} u\right)\left(k_{1} \ldots k_{n-1}\right)
\end{aligned}
$$


Therefore for $1 \leqslant i \leqslant n$, we have $\partial_{i}^{ \pm} \square_{n}^{-} s_{n} u=\partial_{i}^{ \pm} \square_{n}^{-} t_{n} u$ for any $(n+1)$-morphism $u$.

Suppose the proposition proved for $p<n$ with $n \geqslant 2$ and take a $n$-dimensional morphism $x$. Set $h_{i}^{ \pm}=\Gamma_{n-2}^{-} \partial_{i}^{ \pm} \square_{n-1}^{-} s_{n-1}(x)$ for $1 \leqslant i \leqslant n-2$, and set $h_{i}^{-}=\square_{n-1}^{-} d_{n-1}^{(-)^{i}} x$ and $h_{i}^{+}=\epsilon_{n-1} \partial_{n-1}^{+} \square_{n-1}^{-} s_{n-1}(x)$ for $i \geqslant n-1$. We are going to verify that $\left(h_{i}^{ \pm}\right)$is a fillable $(n-1)$-shell. It is sufficient to prove that for any $i$ and any $j$ between 1 and $n$, and any $\alpha, \beta \in\{-,+\}$, the equality $\partial_{i}^{\alpha} h_{j}^{\beta}=\partial_{j-1}^{\beta} h_{i}^{\alpha}$ holds as soon as $1 \leqslant i<j \leqslant n$.

First treat the case $i<j \leqslant n-2$. We have

$$
\begin{aligned}
\partial_{i}^{\alpha} h_{j}^{\beta} & =\partial_{i}^{\alpha} \Gamma_{n-2}^{-} \partial_{j}^{\beta} \square_{n-1}^{-} s_{n-1}(x) \text { since } j<n-1 \\
& =\Gamma_{n-3}^{-} \partial_{i}^{\alpha} \partial_{j}^{\beta} \square_{n-1}^{-} s_{n-1}(x) \text { since } i<n-2 \\
& =\Gamma_{n-3}^{-} \partial_{j-1}^{\beta} \partial_{i}^{\alpha} \square_{n-1}^{-} s_{n-1}(x) \\
& =\partial_{j-1}^{\beta} h_{i}^{\alpha} \text { since } i<n-1
\end{aligned}
$$

Now treat the case $i<j=n-1$. We have

$$
\begin{aligned}
\partial_{i}^{ \pm} h_{j}^{-} & =\partial_{i}^{ \pm} \square_{n-1}^{-} d_{n-1}^{(-)^{n-1}} x \\
& =\partial_{j-1}^{-} \Gamma_{n-2}^{-} \partial_{i}^{ \pm} \square_{n-1}^{-} d_{n-1}^{(-)^{n-1}} x \\
& =\partial_{j-1}^{-} h_{i}^{ \pm}
\end{aligned}
$$

We have also

$$
\begin{aligned}
\partial_{i}^{ \pm} h_{n-1}^{+} & =\partial_{i}^{ \pm} \epsilon_{n-1} \partial_{n-1}^{+} \square_{n-1}^{-} s_{n-1}(x) \\
& =\epsilon_{n-2} \partial_{i}^{ \pm} \partial_{n-1}^{+} \square_{n-1}^{-} s_{n-1}(x) \\
& =\epsilon_{n-2} \partial_{n-2}^{+} \partial_{i}^{ \pm} \square_{n-1}^{-} s_{n-1}(x) \\
& =\partial_{n-2}^{+} \Gamma_{n-2}^{-} \partial_{i}^{ \pm} \square_{n-1}^{-} s_{n-1}(x) \\
& =\partial_{n-2}^{+} h_{i}^{ \pm}
\end{aligned}
$$

Now treat the case $i<j=n$ and $i<n-1$. We have

$$
\begin{aligned}
\partial_{i}^{ \pm} h_{n}^{-} & =\partial_{i}^{ \pm} \square_{n-1}^{-} d_{n-1}^{(-)^{n-1}}(x) \\
& =\partial_{n-1}^{-} \Gamma_{n-2}^{-} \partial_{i}^{ \pm} \square_{n-1}^{-} d_{n-1}^{(-)^{n-1}}(x) \\
& =\partial_{n-1}^{-} h_{i}^{ \pm}
\end{aligned}
$$

and

$$
\begin{aligned}
\partial_{i}^{ \pm} h_{n}^{+} & =\partial_{i}^{ \pm} \epsilon_{n-1} \partial_{n-1}^{+} \square_{n-1}^{-} s_{n-1}(x) \\
& =\epsilon_{n-2} \partial_{i}^{ \pm} \partial_{n-1}^{+} \square_{n-1}^{-} s_{n-1}(x) \\
& =\epsilon_{n-2} \partial_{n-2}^{+} \partial_{i}^{ \pm} \square_{n-1}^{-} s_{n-1}(x) \\
& =\partial_{n-1}^{+} \Gamma_{n-2}^{-} \partial_{i}^{ \pm} \square_{n-1}^{-} s_{n-1}(x) \\
& =\partial_{n-1}^{+} h_{i}^{ \pm}
\end{aligned}
$$


Finally treat the case $i=n-1$ and $j=n$. We have

$$
\begin{aligned}
\partial_{n-1}^{-} h_{n}^{-} & =\partial_{n-1}^{-} \square_{n-1}^{-} d_{n-1}^{(-)^{n}}(x) \\
& =\partial_{n-1}^{-} \square_{n-1}^{-} d_{n-1}^{(-)^{n-1}}(x) \\
& =\partial_{n-1}^{-} h_{n-1}^{-} \\
\partial_{n-1}^{-} h_{n}^{+} & =\partial_{n-1}^{-} \epsilon_{n-1} \partial_{n-1}^{+} \square_{n-1}^{-} s_{n-1}(x) \\
& =\partial_{n-1}^{+} \square_{n-1}^{-} s_{n-1}(x) \\
& =\partial_{n-1}^{+} \square_{n-1}^{-} d_{n-1}^{(-)^{n-1}}(x) \\
& =\partial_{n-1}^{+} h_{n-1}^{-} \\
\partial_{n-1}^{+} h_{n}^{-} & =\partial_{n-1}^{+} \square_{n-1}^{-} d_{n-1}^{(-)^{n}}(x) \\
& =\partial_{n-1}^{-} \epsilon_{n-1} \partial_{n-1}^{+} \square_{n-1}^{-} s_{n-1}(x) \\
& =\partial_{n-1}^{-} h_{n-1}^{+} \\
\partial_{n-1}^{+} h_{n}^{+} & =\partial_{n-1}^{+} \epsilon_{n-1} \partial_{n-1}^{+} \square_{n-1}^{-} s_{n-1}(x) \\
& =\partial_{n-1}^{+} h_{n-1}^{+}
\end{aligned}
$$

Corollary 7.3. Let $\mathcal{C}$ be an $\omega$-category and let $n \geqslant 1$. Set $\mathcal{C}_{\leqslant n}=\mathcal{C}_{1} \cup \ldots \cup \mathcal{C}_{n}$ There exists one and only one natural map $\square_{n}^{-}$from $\mathcal{C}_{\leqslant n}$ to $\omega C$ at $\left(I^{n}, \mathcal{C}\right)^{-}$such that the following axioms hold :

1. if $n \geqslant 3$ and $1 \leqslant i \leqslant n-2$, then $\partial_{i}^{ \pm} \square_{n}^{-}=\Gamma_{n-2}^{-} \partial_{i}^{ \pm} \square_{n-1}^{-} s_{n-1}$.

2. if $n \geqslant 2$ and $n-1 \leqslant i \leqslant n$, then $\partial_{i}^{-} \square_{n}^{-}=\square_{n-1}^{-} d_{n-1}^{(-)^{i}}$ and $\partial_{i}^{+} \square_{n}^{-}=\epsilon_{n-1} \partial_{n-1}^{+} \square_{n-1}^{-} s_{n-1}$. Moreover for $1 \leqslant i \leqslant n$, we have $\partial_{i}^{ \pm} \square_{n}^{-} s_{n}=\partial_{i}^{ \pm} \square_{n}^{-} t_{n}$.

Let $D_{*}^{-}(\mathcal{C})$ be the acyclic group complex generated by the degenerate elements of the negative simplicial nerve of $\mathcal{C}$ with the conventions $D_{n}^{-}(\mathcal{C}) \subset \mathbb{Z} \omega \operatorname{Cat}\left(I^{n}, \mathcal{C}\right)$ and $D_{1}^{-}(\mathcal{C})=$ $D_{0}^{-}(\mathcal{C})=0$. We get thus the following proposition :

Proposition 7.4. The natural linear map $h_{n}^{-}$from $\mathbb{Z} \mathcal{C}_{n}$ to $\mathbb{Z} \omega C$ Cat $\left(I^{n}, \mathcal{C}\right)^{-}$which sends $x_{n}$ to $\square_{n}^{-} x_{n}$ for $n \geqslant 1$ and which associates $\left(x_{0}, y_{0}\right) \in \mathbb{Z C}_{0} \times \mathbb{Z} \mathcal{C}_{0}$ to $x_{0} \in \mathbb{Z C}_{0}$ induces a natural complex morphism

$$
h^{-}: C_{*}^{g l}(\mathcal{C}) \longrightarrow \mathbb{Z} \omega \operatorname{Cat}\left(I^{*}, \mathcal{C}\right)^{-} / D_{*}^{-}(\mathcal{C})
$$


Proof. Let $n \geqslant 2$ and let $x_{n} \in \mathbb{Z C}_{n}$. We have to compare $\sum_{j=1}^{j=n}(-1)^{j+1} \partial_{j}^{-} \square_{n}^{-} x_{n}$ and $\square_{n-1}^{-}\left(s_{n-1}\left(x_{n}\right)-t_{n-1}\left(x_{n}\right)\right)$ modulo elements of $D_{*}^{-}(\mathcal{C})$. We get

$$
\begin{aligned}
& \sum_{j=1}^{j=n}(-1)^{j+1} \partial_{j}^{-} \square_{n}^{-} x_{n} \\
& =\sum_{j=1}^{j=n-2}(-1)^{j+1} \Gamma_{n-2}^{-} \partial_{j}^{-} \square_{n-1}^{-} s_{n-1} x_{n}+(-1)^{n} \square_{n-1}^{-}\left(d_{n-1}^{(-)^{n-1}}\left(x_{n}\right)-d_{n-1}^{(-)^{n}}\left(x_{n}\right)\right) \\
& =\square_{n-1}^{-}\left(s_{n-1}\left(x_{n}\right)-t_{n-1}\left(x_{n}\right)\right) \bmod D_{*}^{-}(\mathcal{C})
\end{aligned}
$$

Now let us treat the case $n=1$. Let $x_{1} \in \mathbb{Z} \mathcal{C}_{1}$. We immediately see that $h_{0}^{-}\left(s_{0}\left(x_{1}\right), t_{0}\left(x_{1}\right)\right)$ and $\partial^{-}\left(\square_{1}^{-}\left(x_{1}\right)\right)$ are equal.

Corollary 7.5. The natural linear map $h_{n}^{-}$from $\mathbb{Z} \mathcal{C}_{n}$ to $\mathbb{Z} \omega C$ at $\left(I^{n}, \mathcal{C}\right)^{-}$which sends $x_{n}$ to $\square_{n}^{-} x_{n}$ for $n \geqslant 1$ and which associates $\left(x_{0}, y_{0}\right) \in \mathbb{Z C}_{0} \times \mathbb{Z} \mathcal{C}_{0}$ to $x_{0} \in \mathbb{Z} \mathcal{C}_{0}$ induces a natural linear map from the globular homology to the negative corner homology of $\mathcal{C}$.

Proof. It is due to the fact that for $n \geqslant 2$, the $n$-th homology group of the quotient chain complex $\mathbb{Z} \omega C$ at $\left(I^{*}, \mathcal{C}\right)^{-} / D_{*}^{-}(\mathcal{C})$ is the $(n-1)$-th homology group of the normalized chain complex associated to the corner simplicial nerve of $\mathcal{C}$.

Now let us expose the construction of $h_{n}^{+}$(without proof).

Proposition 7.6. Let $\mathcal{C}$ be an $\omega$-category and let $n \geqslant 1$. Set $\mathcal{C}_{\leqslant n}=\mathcal{C}_{1} \cup \ldots \cup \mathcal{C}_{n}$ There exists one and only one natural map $\square_{n}^{+}$from $\mathcal{C}_{\leqslant n}$ to $\omega$ Cat $\left(I^{n}, \mathcal{C}\right)^{+}$such that the following axioms hold:

1. if $n \geqslant 3$ and $1 \leqslant i \leqslant n-2$, then $\partial_{i}^{ \pm} \square_{n}^{+}=\Gamma_{n-2}^{+} \partial_{i}^{ \pm} \square_{n-1}^{+} s_{n-1}$.

2. if $n \geqslant 2$ and $n-1 \leqslant i \leqslant n$, then $\partial_{i}^{+} \square_{n}^{+}=\square_{n-1}^{+} d_{n-1}^{(-)^{i+1}}$ and $\partial_{i}^{-} \square_{n}^{+}=$ $\epsilon_{n-1} \partial_{n-1}^{+} \square_{n-1}^{+} s_{n-1}$.

Moreover for $1 \leqslant i \leqslant n$, we have $\partial_{i}^{ \pm} \square_{n}^{+} s_{n}=\partial_{i}^{ \pm} \square_{n}^{+} t_{n}$.

Proposition 7.7. The natural linear map $h_{n}^{+}$from $\mathbb{Z} \mathcal{C}_{n}$ to $\mathbb{Z} \omega \operatorname{Cat}\left(I^{n}, \mathcal{C}\right)^{+}$which sends $x_{n}$ to $\square_{n}^{+} x_{n}$ for $n \geqslant 1$ and which sends $\left(x_{0}, y_{0}\right) \in \mathbb{Z} \mathcal{C}_{0} \oplus \mathbb{Z} \mathcal{C}_{0}$ to $y_{0}$ induces a natural complex morphism

$$
h^{+}: C_{*}^{g l}(\mathcal{C}) \longrightarrow \mathbb{Z} \omega \operatorname{Cat}\left(I^{*}, \mathcal{C}\right)^{+} / D_{*}^{+}(\mathcal{C})
$$

where $D_{*}^{+}(\mathcal{C})$ is the sub-complex of $\mathbb{Z} \omega C$ at $\left(I^{*}, \mathcal{C}\right)^{+}$generated by the degenerate elements of the positive corner simplicial nerve. Therefore $h_{n}^{+}$induces a natural linear map from $H_{n}^{g l}(\mathcal{C})$ to $H_{n}^{+}(\mathcal{C})$. 


\section{Toward an "oriented algebraic topology"}

\subsection{Homotopic $\omega$-categories}

Now we want to speculate about the notion of homotopic $\omega$-categories. We proceed like in algebraic topology by defining a notion of homotopy between non 1-contracting $\omega$-functors, and hence we deduce a notion of homotopy equivalence of $\omega$-category. We are obliged to work with non 1-contracting $\omega$-functors because of the globular and corner homologies.

Intuitively, we could say that two non 1-contracting $\omega$-functors $f$ and $g$ from $\mathcal{C}$ to $\mathcal{D}$ are homotopic if $f(\mathcal{C})$ and $g(\mathcal{C})$ have the same "oriented topology". So a first attempt of definition could be : the $\omega$-functors $f$ and $g$ are homotopic if for any $x \in \mathcal{C}, f(x) \sim g(x)$. Unfortunatly, $f(x)$ and $g(x)$ do not have necessarily the same dimension. So this definition does not make sense, except if $x$ is 0 -dimensional : in this case, $f(x) \sim g(x)$ means $f(x)=$ $g(x)$. It is plausible to think that if $f$ and $g$ were homotopic, then $C_{*}^{g l}(f)$ and $C_{*}^{g l}(g)$ would be two chain homotopic morphisms from $C_{*}^{g l}(\mathcal{C})$ to $C_{*}^{g l}(\mathcal{D})$. So we propose this definition :

Definition 8.1. Let $f$ and $g$ be two non 1-contracting $\omega$-functors from an $\omega$-category $\mathcal{C}$ to an $\omega$-category $\mathcal{D}$. The morphisms $f$ and $g$ are homotopic if the following conditions hold:

1. for any 0-dimensional $x$ of $\mathcal{C}$, one has $f(x)=g(x)$.

2. there exists a linear map $h_{1}$ from $\mathbb{Z C}_{1}$ to $\mathbb{Z D}_{2}$ such that $\left(s_{1}-t_{1}\right) h_{1}(x)=f(x)-g(x)$ for any 1-morphism $x$ of $\mathcal{C}$.

3. for any $n \geqslant 2$, there exists a linear map $h_{n}$ from $\mathbb{Z} \mathcal{C}_{n}$ to $\mathbb{Z} \mathcal{D}_{n+1}$ such that for any $n$-morphism $x$ of $\mathcal{C}$, we have

$$
h_{n-1}\left(s_{n-1}-t_{n-1}\right)(x)+\left(s_{n}-t_{n}\right) h_{n}(x)=f(x)-g(x) \bmod \mathbb{Z} \mathcal{D}_{n-1}
$$

We denote this property by $f \sim_{\left(h_{*}\right)} g$ or more simply $f \sim g$ whenever it is not necessary to precise the homotopy map.

Proposition 8.1. The binary relation "is homotopic to" is an equivalence relation on the collection of non 1-contracting $\omega$-functors from a given $\omega$-category $\mathcal{C}$ to a given $\omega$-category D.

Proof. One has $f \sim f$ since $f \sim_{(0)} f$. If $f \sim_{\left(h_{*}\right)} g$, then $g \sim_{\left(-h_{*}\right)} f$. Now suppose that $f \sim_{\left(h_{*}^{1}\right)} g$ and $g \sim_{\left(h_{*}^{2}\right)} k$. Then $f \sim_{\left(h_{*}^{1}+h_{*}^{2}\right)} k$. 
Proposition 8.2. The homotopy equivalence of non 1-contracting $\omega$-functors is compatible with the composition of non 1-contracting $\omega$-functors in the following sense. Take a diagram in $\omega$ Cat $_{1}$

$$
\mathcal{C} \stackrel{f}{\longrightarrow} \mathcal{D} \stackrel{g}{\stackrel{g}{\longrightarrow}} \mathcal{E} \stackrel{k}{\longrightarrow} \mathcal{F}
$$

If $g \sim h$, then $g \circ f \sim h \circ f, k \circ g \sim k \circ h$ and $k \circ g \circ f \sim k \circ h \circ f$.

Proof. Suppose that $g \sim_{H_{*}} h$. Define $H^{f}$ like this $(n \geqslant 1)$

1. if $x \in \mathcal{C}_{n}$ and if $\operatorname{dim}(f(x))<n$, then $H_{n}^{f}(x):=0$

2. if $x \in \mathcal{C}_{n}$ and if $\operatorname{dim}(f(x))=n$, then $H_{n}^{f}(x):=H_{n}(f(x))$.

If $x$ is 1-dimensional, then

$$
\begin{aligned}
& g \circ f(x)-h \circ f(x) \\
& =\left(s_{1}-t_{1}\right) H_{1}(f(x)) \text { since } f \text { is non 1-contracting } \\
& =\left(s_{1}-t_{1}\right) H_{1}^{f}(x)
\end{aligned}
$$

If $x$ is of dimension $n$ greater than 2 , then either $\operatorname{dim}(f(x))<n$ and in this case

$$
\begin{aligned}
& g \circ f(x)-h \circ f(x) \bmod \mathbb{Z} \mathcal{E}_{n-1} \\
= & 0 \\
= & H_{n-1}\left(s_{n-1}-t_{n-1}\right)(f(x))+\left(s_{n}-t_{n}\right) H_{n}^{f}(x) \\
= & H_{n-1} \circ f \circ\left(s_{n-1}-t_{n-1}\right)(x)+\left(s_{n}-t_{n}\right) H_{n}^{f}(x) \text { since } f \text { is an } \omega \text {-functor } \\
= & H_{n-1}^{f}\left(s_{n-1}-t_{n-1}\right)(x)+\left(s_{n}-t_{n}\right) H_{n}^{f}(x)
\end{aligned}
$$

since $H_{n-1} \circ f \circ\left(s_{n-1}-t_{n-1}\right)(x)=0$. Or $\operatorname{dim}(f(x))=n$ and in that case

$$
\begin{aligned}
& g \circ f(x)-h \circ f(x) \bmod \mathbb{Z} \mathcal{E}_{n-1} \\
= & H_{n-1}\left(s_{n-1}-t_{n-1}\right)(f(x))+\left(s_{n}-t_{n}\right) H_{n}^{f}(x) \\
= & H_{n-1} \circ f \circ\left(s_{n-1}-t_{n-1}\right)(x)+\left(s_{n}-t_{n}\right) H_{n}^{f}(x) \text { since } f \text { is an } \omega \text {-functor }
\end{aligned}
$$

Since $f(x)$ is $n$-dimensional, then $f\left(s_{n-1}(x)\right)=s_{n-1} \circ f(x)$ and $f\left(t_{n-1}(x)\right)=t_{n-1} \circ f(x)$ are $(n-1)$-dimensional. Therefore

$$
g \circ f(x)-h \circ f(x) \bmod \mathbb{Z} \mathcal{E}_{n-1}=H_{n-1}^{f}\left(s_{n-1}-t_{n-1}\right)(x)+\left(s_{n}-t_{n}\right) H_{n}^{f}(x)
$$

Therefore $g \circ f \sim_{H^{f}} h \circ f$. 
Now define ${ }^{k} H$ by ${ }^{k} H_{n}(x):=k\left(H_{n}(x)\right)$ for $x \in \mathcal{C}_{n}$. Then for $x$ 1-dimensional, we have

$$
\begin{aligned}
& k \circ g(x)-k \circ h(x) \\
= & k \circ\left(s_{1}-t_{1}\right) H_{1}(x) \\
= & \left(s_{1}-t_{1}\right)^{k} H_{1}(x) \text { since } k \text { is an } \omega \text {-functor }
\end{aligned}
$$

And for $x$ of dimension greater than 2, we have

$$
\begin{aligned}
& k \circ g(x)-k \circ h(x) \bmod \mathbb{Z} \mathcal{F}_{n-1} \\
= & k\left(H_{n-1}\left(s_{n-1}-t_{n-1}\right)(x)+\left(s_{n}-t_{n}\right) H_{n}(x)\right) \\
= & { }^{k} H_{n-1}\left(s_{n-1}-t_{n-1}\right)(x)+\left(s_{n}-t_{n}\right){ }^{k} H_{n}(x) \text { since } k \text { is an } \omega \text {-functor }
\end{aligned}
$$

Therefore $k \circ g \sim_{{ }_{H} H_{*}} k \circ h$.

Proposition 8.3. Let $f$ and $g$ be two non 1-contracting $\omega$-functors such that for any $x$ of dimension lower than $n, f(x)$ and $g(x)$ are two homotopic dim $(x)$-dimensional morphisms. Then $f$ and $g$ are homotopic as $n$-functor from $\tau_{n} \mathcal{C}$ to $\tau_{n} \mathcal{D}$ (when we consider only the morphisms of dimension lower than n). In other terms, the "oriented topology" is the same in dimension lower than $n$.

Proof. For any $x$ of dimension $1 \leqslant d \leqslant n$, there exists $h_{d}(x) \in \mathbb{Z} \mathcal{D}_{d+1}$ such that $\left(s_{d}-\right.$ $\left.t_{d}\right)\left(h_{d}(x)\right)=f(x)-g(x)$. By convention, we take $h_{d}(x)=0$ whenever $f(x)=g(x)$. However $s_{d-1}(f(x)-g(x))=s_{d-1}\left(s_{d}-t_{d}\right)\left(h_{d}(x)\right)=0$ and in the same way, we have $t_{d-1}(f(x)-g(x))=t_{d-1}\left(s_{d}-t_{d}\right)\left(h_{d}(x)\right)=0$. Therefore $h_{d-1}\left(s_{d-1} x\right)=h_{d-1}\left(t_{d-1} x\right)=0$ and

$$
h_{d-1}\left(s_{d-1}-t_{d-1}\right)(x)+\left(s_{d}-t_{d}\right) h_{d}(x)=f(x)-g(x)
$$

Definition 8.2. Let $\mathcal{C}$ and $\mathcal{D}$ be two $\omega$-categories. They are homotopic if and only if there exists a non 1-contracting $\omega$-functor $f$ from $\mathcal{C}$ to $\mathcal{D}$ and a non 1-contracting $\omega$-functor $g$ from $\mathcal{D}$ to $\mathcal{C}$ such that $f \circ g \sim I d_{\mathcal{D}}$ and $g \circ f \sim I d_{\mathcal{C}}$. We say that $f$ and $g$ are homotopy equivalences between the two $\omega$-categories $\mathcal{C}$ and $\mathcal{D}$.

Proposition 8.4. The homotopy equivalence is an equivalence relation indeed on the collection of $\omega$-categories.

Proof. This relation is obviously reflexible and symmetric. It remains to prove the transitivity. Let us consider the following diagram in $\omega \mathrm{Cat}_{1}$ :

$$
\mathcal{C} \stackrel{f}{\stackrel{f}{\rightleftarrows}} \mathcal{D} \underset{k}{\stackrel{h}{\rightleftarrows}} \mathcal{E}
$$


and suppose that $g \circ f \sim I d_{\mathcal{C}}, f \circ g \sim I d_{\mathcal{D}}, h \circ k \sim I d_{\mathcal{E}}$ and $k \circ h \sim I d_{\mathcal{D}}$. Then $g \circ k \circ h \circ f \sim g \circ f \sim I d_{\mathcal{C}}$ and $h \circ f \circ g \circ k \sim h \circ k \sim I d_{\mathcal{E}}$.

Now some examples of homotopic $\omega$-categories.

Proposition 8.5. For any natural number $p \geqslant 1$ and $q \geqslant 1,2_{p}$ (the free $\omega$-category generated by one p-morphism) and $2_{q}$ are homotopic.

Proof. If $p=q$, it is trivial. Suppose that $p>q$. Let $f$ be the only $\omega$-functor from $\langle A\rangle=2_{p}$ to $\langle B\rangle=2_{q}$ such that $f(A)=B$ and let $g$ be the unique functor from $\langle B\rangle$ to $\langle A\rangle$ such that $g(B)=s_{q}(A)$. Then $f \circ g=I d_{2_{q}}$ so $f \circ g$ and $I d_{2_{q}}$ are homotopic as $\omega$-functors. Now consider $g \circ f$ and $I d_{2_{q}}$. Set

$$
\begin{aligned}
& h_{r}=0 \text { if } 1 \leqslant r<q \\
& h_{r}\left(s_{r} A\right)=0 \text { and } h_{r}\left(t_{r} A\right)=s_{r+1} A \text { if } q \leqslant r<p \\
& h_{r}(A)=0 \text { if } r \geqslant p
\end{aligned}
$$

First suppose that $q=1$. Then we have $\left(s_{1}-t_{1}\right) h_{1}\left(s_{1} A\right)=0=(g \circ f)\left(s_{1} A\right)-s_{1} A$ and $\left(s_{1}-t_{1}\right) h_{1}\left(t_{1} A\right)=s_{1} A-t_{1} A=(g \circ f)\left(t_{1} A\right)-t_{1} A$, and for any $1<r<p$, we have

$$
\begin{aligned}
& h_{r-1}\left(s_{r-1}-t_{r-1}\right)\left(s_{r} A\right)+\left(s_{r}-t_{r}\right) h_{r}\left(s_{r} A\right) \\
= & -s_{r} A \\
= & g \circ f\left(s_{r} A\right)-s_{r} A \bmod \left(2_{p}\right)_{r-1}
\end{aligned}
$$

and

$$
\begin{aligned}
& h_{r-1}\left(s_{r-1}-t_{r-1}\right)\left(t_{r} A\right)+\left(s_{r}-t_{r}\right) h_{r}\left(t_{r} A\right) \\
= & -s_{r} A+s_{r} A-t_{r} A \\
= & s_{1} A-t_{r} A \bmod \left(2_{p}\right)_{r-1} \text { since } r>1 \\
= & g \circ f\left(t_{r} A\right)-t_{r} A
\end{aligned}
$$

In order to complete the case $q=1$, now suppose that $r=p$. Then

$$
\begin{aligned}
& h_{r-1}\left(s_{r-1}-t_{r-1}\right)(A)+\left(s_{r}-t_{r}\right) h_{r}(A) \\
= & -A \\
= & s_{1} A-A \bmod \left(2_{p}\right)_{p-1} \text { since } p \geqslant 2 \\
= & g \circ f(A)-A
\end{aligned}
$$

Now suppose that $q>1$. The different cases may be treated in the same way. First set $r=1$. Then $\left(s_{1}-t_{1}\right) h_{1}\left(s_{1} A\right)=0=(g \circ f)\left(s_{1} A\right)-s_{1} A$ and $\left(s_{1}-t_{1}\right) h_{1}\left(t_{1} A\right)=s_{1} A-t_{1} A=$ 
$(g \circ f)\left(t_{1} A\right)-t_{1} A$, and for any $1<r<q$, we have

$$
\begin{aligned}
& h_{r-1}\left(s_{r-1}-t_{r-1}\right)\left(s_{r} A\right)+\left(s_{r}-t_{r}\right) h_{r}\left(s_{r} A\right) \\
= & 0 \\
= & g \circ f\left(s_{r} A\right)-s_{r} A
\end{aligned}
$$

and

$$
\begin{aligned}
& h_{r-1}\left(s_{r-1}-t_{r-1}\right)\left(t_{r} A\right)+\left(s_{r}-t_{r}\right) h_{r}\left(t_{r} A\right) \\
= & 0 \\
= & g \circ f\left(t_{r} A\right)-t_{r} A
\end{aligned}
$$

For $r=q$, we have

$$
\begin{aligned}
& h_{q-1}\left(s_{q-1}-t_{q-1}\right)\left(s_{q} A\right)+\left(s_{q}-t_{q}\right) h_{q}\left(s_{q} A\right) \\
= & 0 \\
= & g \circ f\left(s_{q} A\right)-s_{q} A
\end{aligned}
$$

and

$$
\begin{aligned}
& h_{q-1}\left(s_{q-1}-t_{q-1}\right)\left(t_{q} A\right)+\left(s_{q}-t_{q}\right) h_{q}\left(t_{q} A\right) \\
= & s_{q} A-t_{q} A \\
= & g \circ f\left(t_{q} A\right)-t_{q} A
\end{aligned}
$$

For $q<r<p$, we have

$$
\begin{aligned}
& h_{r-1}\left(s_{r-1}-t_{r-1}\right)\left(s_{r} A\right)+\left(s_{r}-t_{r}\right) h_{r}\left(s_{r} A\right) \\
= & -s_{r} A \\
= & s_{q} A-s_{r} A \bmod \left(2_{p}\right)_{r-1} \text { since } q \leqslant r-1 \\
= & g \circ f\left(s_{r} A\right)-s_{r} A
\end{aligned}
$$

and

$$
\begin{aligned}
& h_{r-1}\left(s_{r-1}-t_{r-1}\right)\left(t_{r} A\right)+\left(s_{r}-t_{r}\right) h_{r}\left(t_{r} A\right) \\
= & -s_{r} A+s_{r} A-t_{r} A \\
= & s_{q} A-t_{r} A \bmod \left(2_{p}\right)_{r-1} \text { since } q \leqslant r-1 \\
= & g \circ f\left(t_{r} A\right)-t_{r} A \bmod \left(2_{p}\right)_{r-1}
\end{aligned}
$$


It remains the case $r=p$ :

$$
\begin{aligned}
& h_{p-1}\left(s_{p-1}-t_{p-1}\right)(A)+\left(s_{p}-t_{p}\right) h_{p}(A) \\
= & -A \\
= & s_{q} A-A \bmod \left(2_{p}\right)_{p-1} \text { since } q \leqslant p-1 \\
= & g \circ f(A)-A
\end{aligned}
$$

Definition 8.3. Let $\mathcal{C}$ be an $\omega$-category. Let $I$ and $F$ be some sets of 0 -morphims of $\mathcal{C}$. The bilocalization of $\mathcal{C}$ with respect to $I$ and $F$ the sub-category of $\mathcal{C}$ consists of the $n$-morphims $f$ such that $s_{0} f \in I$ and $t_{0} f \in F$ with the induced structure of $\omega$-category. This $\omega$-category is denoted by $\mathcal{C}(I, F)$.

In the sequel, the set $I$ will be always the set of initial states and the set $F$ the set of final states of the considered $\omega$-category.

Now we prove that the bilocalization of $\omega$-category $\mathcal{C}$ of Figure 1 with respect to $I$ (the intersection of $u$ and $e$ ) and $F$ (the intersection of $z$ and $d$ ) is homotopic to the $\omega$-category of Figure 0, denoted by $G_{1}[A, B]$. Let $f$ be the unique $\omega$-functor from $\mathcal{C}\left(s_{0}(u), t_{0}(z)\right)$ to $G_{1}[A, B]$ which maps any 1-path homotopic to $\gamma_{1}$ to $A$ and any 1-path homotopic to $\gamma_{4}$ to $B$. Let $g$ be the unique $\omega$-functor from $G_{1}[A, B]$ to $\mathcal{C}\left(s_{0}(u), t_{0}(z)\right)$ which maps $A$ to $\gamma_{1}$ and $B$ to $\gamma_{4}$. Then $f \circ g=I d_{G_{1}[A, B]}$. It remains to prove that $g \circ f$ and $I d_{\mathcal{C}\left(s_{0}(u), t_{0}(z)\right)}$ are homotopic $\omega$-functors. For any 1-morphism $x$ of $\mathcal{C}\left(s_{0}(u), t_{0}(z)\right), g \circ f(x)$ and $x$ are homotopic 1-morphisms. Let $h_{1}(x)$ be the element of $\mathbb{Z C}\left(s_{0}(u), t_{0}(z)\right)_{2}$ such that $\left(s_{1}-t_{1}\right) h_{1}(x)=$ $g \circ f(x)-x$. Take $h_{2}=0$. We have to verify that for any 2 -morphism $C$, we have

$$
h_{1}\left(s_{1}-t_{1}\right) C+\left(s_{2}-t_{2}\right) h_{2} C=g \circ f(C)-C \bmod \mathcal{C}\left(s_{0}(u), t_{0}(z)\right)_{1} .
$$

Suppose for example that $s_{1} C$ is homotopic to $\gamma_{1}$. Then $h_{1} s_{1} C$ is the unique element of $\mathbb{Z C}\left(s_{0}(u), t_{0}(z)\right)_{2}$ such that $\left(s_{1}-t_{1}\right) h_{1} s_{1} C=\gamma_{1}-s_{1} C$. And $h_{1} t_{1} C$ is the unique element of $\mathbb{Z C}\left(s_{0}(u), t_{0}(z)\right)_{2}$ such that $\left(s_{1}-t_{1}\right) h_{1} t_{1} C=\gamma_{1}-t_{1} C$. Then $h_{1}\left(s_{1}-t_{1}\right) C$ is the unique element of $\mathbb{Z C}\left(s_{0}(u), t_{0}(z)\right)_{2}$ such that $\left(s_{1}-t_{1}\right) h_{1}\left(s_{1}-t_{1}\right) C=t_{1} C-s_{1} C$. Therefore $h_{1}\left(s_{1}-\right.$ $\left.t_{1}\right) C=-C$. Hence the result.

Now here are some other examples without proof. In Figure 8, the bilocalization of the depicted $\omega$-categories with respect to their set of initial and final states are homotopic.

Figure 19 represents a cubical set with a 3-dimensional cubical hole. Then the bilocalization of this 3 -category with respect to its sets of initial and final states is homotopic to $G_{2}[A, B]$, the 2-category of Figure 2 generated by two non homotopic 2-morphisms $A$ and $B$ having the same 1-source and the same 1-target. 


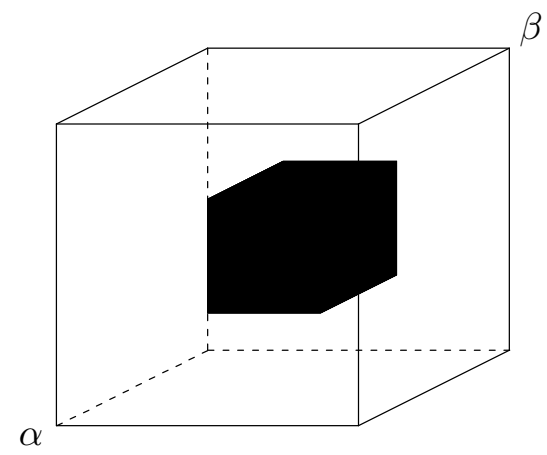

Figure 19: A 2-semaphore

\subsection{Invariance of the globular and corner homologies}

Theorem 8.6. Let $f$ and $g$ be two non 1-contracting $\omega$-functors from $\mathcal{C}$ to $\mathcal{D}$. Suppose that $f$ and $g$ are homotopic. Then for all natural number $n, f$ and $g$ induce linear maps from $H_{n}^{g l}(\mathcal{C})$ to $H_{n}^{g l}(\mathcal{D})$ and moreover $H_{n}^{g l}(f)=H_{n}^{g l}(g)$.

Proof. Take two homotopic $\omega$-functors $f$ and $g$. Let $x_{1}$ be a globular 1-cycle. Then $(f-$ $g)\left(x_{1}\right)=\left(s_{1}-t_{1}\right) h_{1}(x)$. Therefore $(f-g)\left(x_{1}\right)=\partial\left(h_{1}(x)\right)$. Now take a globular $n$-cycle $x_{n}$ with $n \geqslant 2$. Then $s_{n-1} x_{n}=t_{n-1} x_{n}$. Therefore $f\left(x_{n}\right)-g\left(x_{n}\right)=\left(s_{n}-t_{n}\right) h_{n} x_{n} \bmod \mathcal{D}_{n-1}$.

The analogous statement for the corner homologies is still a conjecture only proved in the following particular case (see Proposition 8.3) :

Theorem 8.7. Let $f$ and $g$ be two non 1 -contracting $\omega$-functor from $\mathcal{C}$ to $\mathcal{D}$. Suppose that for any $x$ of dimension strictly lower than $n, f(x)=g(x)$ and such that for $x$-dimensional, $f(x)$ and $g(x)$ are two homotopic $n$-morphisms of $\mathcal{D}$. Then for any $p \leqslant n, f_{p}$ (resp. $\left.g_{p}\right)$ yield linear maps from $H_{p}^{\alpha}(\mathcal{C})$ to $H_{p}^{\alpha}(\mathcal{D})$ for $\alpha \in\{-,+\}$ and moreover, $H_{p}^{\alpha}(f)=H_{p}^{\alpha}(g)$.

Proof. We make the proof for $\alpha=-$. The homology of the non normalized complex associated to a simplicial group is equal to its homotopy (Wei94 Theorem 8.3.8). Therefore it suffices to find an homotopy between $f(x)$ and $g(x)$ in $\mathcal{N}^{-}(\mathcal{C})$ for any $x \in \omega \operatorname{Cat}\left(I^{n}, \mathcal{C}\right)$. We can suppose without loss of generality that there exists a $(n+1)$-dimensional morphism $u$ of $\mathcal{C}$ such that $s_{n}(u)=f(x)\left(0_{n}\right)$ and $t_{n}(u)=g(x)\left(0_{n}\right)$. Then consider the following realizations $h_{i}^{ \pm}$of $I^{n}$ for $1 \leqslant i \leqslant n+1$ : for $i$ between 1 and $n-1, h_{i}^{ \pm}=\Gamma_{n-1}^{-} \partial_{i}^{ \pm} x=\Gamma_{n-1}^{-} \partial_{i}^{ \pm} y$, $h_{n}^{-}=d_{n}^{\epsilon}(u), h_{n+1}^{-}=d_{n}^{\epsilon+1}(u)$ with $\epsilon$ equal to 0 or 1 depending on the parity of $n$, and finally $h_{n}^{+}=h_{n+1}^{+}=\epsilon_{n} \partial_{n}^{+} x=\epsilon_{n} \partial_{n}^{+} y$. We obtain the fillable $n$-shell which already appears in Proposition 7.2. The corresponding element of $\omega C a t\left(I^{n+1}, \mathcal{C}\right)$ yields an homotopy in $\mathcal{N}^{-}(\mathcal{C})$ between $f(x)$ and $g(x)$. 


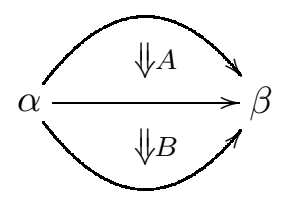

Figure 20: Composition of two 2-morphims

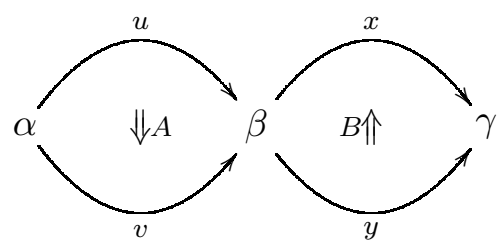

Figure 21: Two 0-composable 2-morphisms

Conjecture 8.8. Let $f$ and $g$ be two non 1-contracting $\omega$-functors from $\mathcal{C}$ to $\mathcal{D}$. Suppose that $f$ and $g$ are homotopic. Then for all natural number $n, f$ and $g$ induce linear maps from $H_{n}^{ \pm}(\mathcal{C})$ to $H_{n}^{ \pm}(\mathcal{D})$ and moreover $H_{n}^{ \pm}(f)=H_{n}^{ \pm}(g)$.

\section{Some open questions and perspectives}

\subsection{Some interesting problems in mathematics}

First of all, we come back to the globular homology of an $\omega$-category. We propose here a small modification of its definition. Consider the $\omega$-category $\mathcal{C}$ of Figure 20 where $A$ and $B$ are two 2-morphisms which are supposed to be composable. Then $H_{2}^{g l}(\mathcal{C}) \neq 0$ since $C_{3}^{g l}(\mathcal{C})=0$ and since $\left(s_{1}-t_{1}\right)\left(A *_{1} B-A-B\right)=0$. However, this globular 2cycle corresponds to nothing real in $\mathcal{C}$. As consequence of this small calculation, we obtain that $H_{2}^{g l}\left(I^{3}\right) \neq 0$ (cf Figure 13). It suffices to consider for example the globular 2-cycle $C *_{1} D-C-D$ with $C=R(-00) *_{0} R(0++)$ and $D=R(-0-) *_{0} R(0+0)$.

Consider the $\omega$-category of Figure 21 where $\alpha, \beta$ and $\gamma$ are 0 -morphisms, $u, v, x$ and 


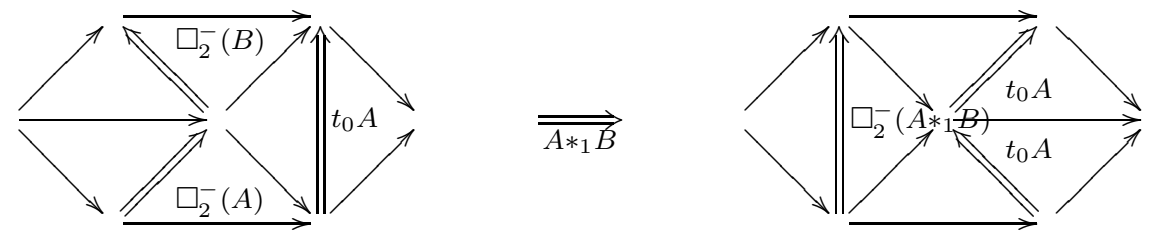

Figure 22: $h_{2}^{-}\left(A *_{1} B-A-B\right)$ is a boundary

$y$ are 1-morphisms and $A$ and $B$ two 2-morphims. There are at least two elements of $\mathbb{Z} \mathcal{C}_{2}$ between $u *_{0} x$ and $v *_{0} y: A *_{0} y-u *_{0} B, A *_{0} x-v *_{0} B$. Therefore $A *_{0} y-u *_{0} B-A *_{0} x+v *_{0} B$ is a globular 2-cycle which means nothing geometrically.

So we propose to modify the definition of the globular homology as follows. Let $\widehat{\omega C a t_{1}}$ be the category whose objects are globular $\omega$-categories such that any $(n+1)$-morphism $X$ is invertible with respect to $*_{n}$ as soon as $n \geqslant 1$ (i.e. there exists a $(n+1)$-morphism $X^{-1}$ such that $\left.s_{n} X^{-1}=t_{n} X, t_{n} X^{-1}=s_{n} X, X *_{n} X^{-1}=s_{n} X, X^{-1} *_{n} X=t_{n} X\right)$ and whose morphisms are non 1-contracting $\omega$-functors. Let us denote by $\mathcal{C} \mapsto \widehat{\mathcal{C}}$ the left adjoint functor to the forgetful functor from $\widehat{\omega C a t_{1}}$ to $\omega C a t_{1}$. We set $C_{0}^{g l}(\mathcal{C})=\mathbb{Z} \mathcal{C}_{0} \oplus \mathbb{Z} \mathcal{C}_{0}$, $C_{1}^{g l}(\mathcal{C})_{1}=\mathbb{Z} \mathcal{C}_{1}$, and $C_{n}^{g l}(\mathcal{C})$ for $n \geqslant 2$ is the free abelian group generated by $\widehat{\mathcal{C}}_{n}$ quotiented by the relations $A+B=A *_{n-1} B \bmod \mathbb{Z C}_{n-1}$ if $A$ and $B$ are two $n$-morphisms such that $t_{n-1} A=s_{n-1} B$. With the same differential map, we obtain a new globular homology theory. Let us denote it by $H_{*}^{\text {new }}-g l$.

With this new homology theory, the above problems disappear. It is obvious for the first one and concerning the second one, here is the reason. In $C_{2}^{\text {new-gl }}(\mathcal{C})$ one has

$$
\begin{aligned}
A *_{0} x-v *_{0} B & =A *_{0} x+\left(v *_{0} B\right)^{-1} \\
& =A *_{0} x+v *_{0} B^{-1} \\
& =\left(A *_{0} x\right) *_{1}\left(v *_{0} B^{-1}\right) \\
& =\left(A *_{1} v\right) *_{0}\left(x *_{1} B^{-1}\right) \\
& =A *_{0} B^{-1} \\
& =\left(u *_{1} A\right) *_{0}\left(B^{-1} *_{1} y\right) \\
& =\left(u *_{0} B^{-1}\right) *_{1}\left(A *_{0} y\right) \\
& =u *_{0} B^{-1}+A *_{0} y \\
& =\left(u *_{0} B\right)^{-1}+A *_{0} y \\
& =A *_{0} y-u *_{0} B
\end{aligned}
$$

It suffices to consider the labelled 3 -cube of Figure 22 to see that $h_{2}^{-}\left(A *_{1} B-A-B\right)$ 
is a boundary in the negative corner homology. In the same way, we can prove that $h_{2}^{+}\left(A *_{1} B-A-B\right)$ is also a boundary, this time in the posivite corner homology. There is a canonical linear map $H_{*}^{g l}(\mathcal{C}) \longrightarrow H_{*}^{n e w-g l}(\mathcal{C})$. Therefore there exists at least for $n=0,1,2$ a natural linear map $\widehat{h}_{n}^{ \pm}$such that the following diagram commutes :

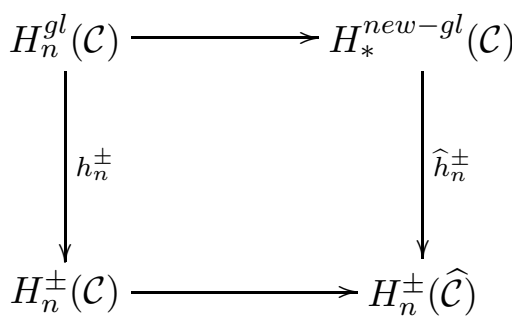

We are led to the following conjectures :

Conjecture 9.1. $\quad$ 1. The group $H_{p}^{\text {new-gl }}\left(I^{n}\right)$ vanishes for every natural number $n$ and any $p>0$.

2. For every $n \geqslant 2$, if $A$ and $B$ are two $n$-morphisms such that $t_{n-1} A=s_{n-1} B$, then $\square_{n}^{ \pm}\left(A *_{n-1} B-A-B\right)$ is a boundary in the corresponding corner homology of $\widehat{\mathcal{C}}$.

3. The canonical map $H_{n}^{ \pm}(\mathcal{C}) \longrightarrow H_{n}^{ \pm}(\widehat{\mathcal{C}})$ is an isomorphism for every $n \geqslant 0$

4. As consequence of the above two conjectures, $h_{n}^{ \pm}$factorizes through the new globular homology theory.

This new definition of the globular homology gives rise to a new definition of homotopic non 1-contracting $\omega$-functors and gives rise to the conjecture that the corner homology theories are still invariant with respect to this new equivalence relation.

Elements like $A *_{1} B-A-B$ could be called thin globular cycles. On the corner homologies, the analogous elements are the linear combinations of $x \in \omega \operatorname{Cat}\left(I^{n}, \mathcal{C}\right)$ for some given natural number $n$ such that $x\left(0_{n}\right)$ is of dimension strictly lower than $n$. We have therefore the following conjecture :

Conjecture 9.2. (About the thin elements of the corner complexes of a free globular $\omega$ category $\mathcal{C})$ Let $x_{i}$ be elements of $\omega C$ Cat $\left(I^{n}, \mathcal{C}\right)^{ \pm}$and let $\lambda_{i}$ be natural numbers, where $i$ runs over some set $I$. Suppose that for any $i, x_{i}\left(0_{n}\right)$ is of dimension strictly lower than $n$. Then $\sum_{i} \lambda_{i} x_{i}$ is a boundary if and only if it is a cycle.

With these new facts about the thin elements, Conjecture 8.8 becomes

Conjecture 9.3. Let $f$ and $g$ be two homotopic non 1-contracting $\omega$-functors from $\mathcal{C}$ to $\mathcal{D}$ where $\mathcal{C}$ and $\mathcal{D}$ are two free $\omega$-categories. Let $x$ be an element of $\omega \operatorname{Cat}\left(I^{n}, \mathcal{C}\right)^{ \pm}$. Then $f(x)-g(x)=B+T$ where $B$ is a boundary of $\mathbb{Z} \omega C a t\left(I^{n}, \mathcal{D}\right)^{ \pm}$and $T$ a linear combination of thin elements of $\mathbb{Z} \omega \operatorname{Cat}\left(I^{n}, \mathcal{D}\right)^{ \pm}$. 


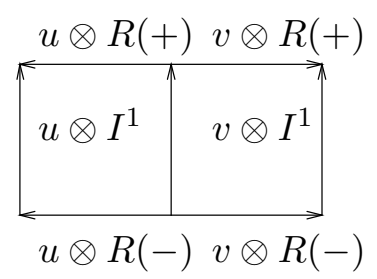

Figure 23: Filling of corners

There exists a unique biclosed monoidal structure $\otimes$ on $\omega C$ at such that $I^{m} \otimes I^{n}=I^{m+n}$. See for example Cra95 for an explicit construction using globular pasting scheme theory. The effect of the functor $-\otimes I^{1}$ is to fill corners as it is showed in Figure 23. We are led to the following conjectures.

Conjecture 9.4. If $\mathcal{C}$ is an $\omega$-category, then the $\omega$-functor from $\mathcal{C}$ to $I^{1} \otimes \mathcal{C}$ which maps $u$ to $R(\mp) \otimes u$ yield an isomorphism from $H_{*}^{ \pm}(\mathcal{C})$ to $H_{*}^{ \pm}\left(I^{1} \otimes \mathcal{C}\right)$ and the zero map from $H_{*}^{\mp}(\mathcal{C})$ to $H_{*}^{\mp}\left(I^{1} \otimes \mathcal{C}\right)$ for $*>0$.

We can easily check that $u \mapsto R(\mp) \otimes u$ induces 0 from $H_{1}^{\mp}(\mathcal{C})$ to $H_{1}^{\mp}\left(I^{1} \otimes \mathcal{C}\right)$. We end up this section with three other problems and one remark about the corner homologies. The conjectures are easy to verify in lower dimension.

Conjecture 9.5. 1. The corner homology groups of $I^{n}$ vanish in dimension strictly greater than 0 . In other terms, if $p>0$, then $H_{p}^{ \pm}\left(I^{n}\right)=0$

2. Let $2_{n}$ be the free $\omega$-category generated by a n-morphism. Then $p>0$ implies $H_{p}^{ \pm}\left(2_{n}\right)=0$

3. Let $G_{n}$ be the oriented $n$-globe with $n>0$, i.e. the free $\omega$-category generated by two non homotopic $n$-morphisms having the same $(n-1)$-source and the same $(n-1)$ target. Then $H_{p}^{ \pm}\left(G_{n}\right)=0$ if $p \neq n$ and $p>0$. Moreover the equality $H_{n}^{ \pm}\left(G_{n}\right)=\mathbb{Z}$ holds.

All the conjectures of this section will be the subject of future papers.

\subsection{Perspectives in computer science}

The study of the cokernel of the negative Hurewicz morphism would allow us to detect the deadlock in concurrent machines. In an analogous way the cokernel of the positive Hurewicz morphism would allow us to detect the unreachable states in a concurrent machine. This is useful for detecting the dead code in a concurrent machines and for analyzing the safety 
properties of a machine [GW91] : proving that a property is false is equivalent to proving that some states are unreachable.

We exhibited in Figure 8 a 1-category homotopic to a $\omega$-category. The 1-category we obtain suggests some relations with the graph of oriented connected components introduced in [FGR98a].

We think also that some problems of confidentiality in computer science involve the construction of a relative corner homology. The problem stands as follows : take a concurrent machine with a flow of inputs and a flow of outputs, every input and output having a confidentiality level ; such a machine is confidential if the flow of inputs of confidentiality level lower than $l$ determines the flow of outputs of confidentiality level $l$ (otherwise an observer could deduce from observations of outputs of confidentiality levels $l$ some information about inputs of confidentiality level greater than $l$ ). The geometric problem which arises from this situation stands as follows : if some $n$-transitions are the inputs and some other ones are the outputs, the problem is to know whether inputs determine outputs over the set of all possible execution paths of the machine. In the 1-dimensional case, using bicomplexes, we already found out a relation between this problem and the vertical and horizontal $H_{1}$ and we suspect that in higher dimension this problem is related in some way with the relative oriented Hurewicz morphisms Gau97a Gau97b Gau97c].

\section{Direct construction of the globular and corner homologies of a cubical set}

In this last section we explain how to obtain the globular and corner homologies of a cubical set by using the free cubical $\omega$-category generated by it, instead of considering the free globular one. This approach could be useful in an algorithmic viewpoint.

\subsection{Cubical $\omega$-category}

The notion of cubical $\omega$-category appears in the (already cited) works of Brown, Higgins, Al-Agl.

Definition 10.1. A cubical $\omega$-category consists of a cubical set with connections

$$
\left(\left(K_{n}\right)_{n \geqslant 0}, \partial_{i}^{\alpha}, \epsilon_{i}, \Gamma_{i}^{\alpha}\right)
$$

together with a family of associative operations $+_{j}$ defined on $\left\{(x, y) \in K_{n} \times K_{n}, \partial_{i}^{+} x=\right.$ $\left.\partial_{i}^{-} y\right\}$ for $1 \leqslant j \leqslant n$ such that

1. $\left(x+{ }_{j} y\right)+{ }_{j} z=x+{ }_{j}\left(y+{ }_{j} z\right)$

2. $\partial_{j}^{-}\left(x+{ }_{j} y\right)=\partial_{j}^{-}(x)$ 
3. $\partial_{j}^{+}\left(x+{ }_{j} y\right)=\partial_{j}^{+}(y)$

4. $\partial_{i}^{\alpha}\left(x+{ }_{j} y\right)=\left\{\begin{array}{l}\partial_{i}^{\alpha}(x)+{ }_{j-1} \partial_{i}^{\alpha}(y) \text { if } i<j \\ \partial_{i}^{\alpha}(x)+{ }_{j} \partial_{i}^{\alpha}(y) \text { if } i>j\end{array}\right.$

5. $\left(x+{ }_{i} y\right)+_{j}\left(z+_{i} t\right)=\left(x+{ }_{j} z\right)+_{i}\left(y+{ }_{j} t\right)$. We will denoted the two members of this equality by

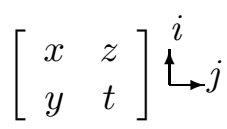

6. $\epsilon_{i}\left(x+{ }_{j} y\right)=\left\{\begin{array}{l}\epsilon_{i}(x)+{ }_{j+1} \epsilon_{i}(y) \text { if } i \leqslant j \\ \epsilon_{i}(x)+{ }_{j} \epsilon_{i}(y) \text { if } i>j\end{array}\right.$

7. $\Gamma_{i}^{ \pm}\left(x+{ }_{j} y\right)=\left\{\begin{array}{l}\Gamma_{i}^{ \pm}(x)+{ }_{j+1} \Gamma_{i}^{ \pm}(y) \text { if } i<j \\ \Gamma_{i}^{ \pm}(x)+{ }_{j} \Gamma_{i}^{ \pm}(y) \text { if } i>j\end{array}\right.$

8. If $i=j, \Gamma_{i}^{-}\left(x+{ }_{j} y\right)=\left[\begin{array}{cc}\epsilon_{j+1}(y) & \Gamma_{j}^{-}(y) \\ \Gamma_{j}^{-}(x) & \epsilon_{j}(y)\end{array}\right]^{j} j+1$

9. If $i=j, \Gamma_{i}^{+}\left(x+{ }_{j} y\right)=\left[\begin{array}{cc}\epsilon_{j}(x) & \Gamma_{j}^{+}(y) \\ \Gamma_{j}^{+}(x) & \epsilon_{j+1}(x)\end{array}\right] \stackrel{\jmath}{\longleftrightarrow} j+1$

10. $\Gamma_{j}^{+} x+{ }_{j+1} \Gamma_{j}^{-} x=\epsilon_{j} x$ and $\Gamma_{j}^{+} x+{ }_{j} \Gamma_{j}^{-} x=\epsilon_{j+1} x$

11. $\epsilon_{i} \partial_{i}^{-} x+{ }_{i} x=x+{ }_{i} \epsilon_{i} \partial_{i}^{+} x=x$

The corresponding category with the obvious morphisms is denoted by $\infty$ Cat.

Look back again to the cubical singular nerve of a topological space $X$. We can equip it with operations $+_{j}$ as follows :

$$
\left(f+{ }_{j} g\right)\left(x_{1}, \ldots, x_{p}\right)=\left\{\begin{array}{c}
f\left(x_{1}, \ldots, 2 x_{i}, \ldots, x_{p}\right) \text { if } x_{i} \leqslant 1 / 2 \\
g\left(x_{1}, \ldots, 2 x_{i}-1, \ldots, x_{p}\right) \text { if } x_{i} \geqslant 1 / 2
\end{array}\right.
$$

All axioms of cubical $\omega$-categories are satisfied except the associativity axiom.

It turns out that $\omega C a t$ and $\infty C a t$ are equivalent. The 2-dimensional case is solved in [Spe77] (which is followed by [SW83]) and the 3-dimensional case is solved in AA89. Recently Richard Steiner developed the methods of Al-Agl to prove the result in all dimensions, as conjectured in Al-Agl. The corresponding result for groupoids was already known from earlier results of Brown-Higgins [BH81a [BH81b. The category equivalence is realized by the functor $\gamma: \infty C a t \longrightarrow \omega C$ at defined as follows $(G \in \infty C a t)$ : 


$$
(\gamma G)_{n}=\left\{x \in G_{n}, \partial_{j}^{\alpha} x \in \epsilon_{1}^{j-1} G_{n-j} \text { for } 1 \leqslant j \leqslant n, \alpha=0,1\right\}
$$

Using general category theory arguments, one can prove that the forgetful functor $U$ from $\infty$ Cat to Sets $\square^{\text {op }}$ has a left adjoint functor $\rho$ which defines therefore the free cubical $\omega$-category generated by a cubical set. We will see in Section 10 that it can be constructed explicitely by considering the cubical singular complex of the free globular $\omega$-category generated by $K$.

\subsection{The globular and corner homologies of a cubical set}

First of all, take a look at the cubical singular nerve :

Proposition 10.1. Let $\mathcal{C}$ be a globular $\omega$-category. For any strictly positive natural number $n$ and any $j$ between 1 and $n$, there exists one and only one natural map ${ }_{j}{ }_{j}$ from the set of pairs $(x, y)$ of $\mathcal{N}^{\square}(\mathcal{C})_{n} \times \mathcal{N}^{\square}(\mathcal{C})_{n}$ such that $\partial_{j}^{+}(x)=\partial_{j}^{-}(x)$ to the set $\mathcal{N}^{\square}(\mathcal{C})_{n}$ which satisfies the following properties:

$$
\begin{aligned}
& \partial_{j}^{-}\left(x+{ }_{j} y\right)=\partial_{j}^{-}(x) \\
& \partial_{j}^{+}\left(x+{ }_{j} y\right)=\partial_{j}^{+}(x) \\
& \partial_{i}^{\alpha}\left(x+{ }_{j} y\right)=\left\{\begin{array}{c}
\partial_{i}^{\alpha}(x)+{ }_{j-1} \partial_{i}^{\alpha}(y) \text { if } i<j \\
\partial_{i}^{\alpha}(x)+{ }_{j} \partial_{i}^{\alpha}(y) \text { if } i>j
\end{array}\right.
\end{aligned}
$$

Moreover, these operations induce a structure of cubical $\omega$-category on $\mathcal{N}^{\square}(\mathcal{C})$.

Proof. We give only a sketch of proof.

Step 1. First of all, we observe that the functor from $\omega C$ at to the category Sets of sets

$$
\mathcal{C} \mapsto \omega \operatorname{Cat}\left(I^{p}, \mathcal{C}\right) \times_{j} \omega \operatorname{Cat}\left(I^{p}, \mathcal{C}\right)=\left\{(x, y) \in \omega \operatorname{Cat}\left(I^{p}, \mathcal{C}\right) \times \omega \operatorname{Cat}\left(I^{p}, \mathcal{C}\right), \partial_{i}^{+} x=\partial_{i}^{-} y\right\}
$$

is representable. We denote by $I^{p}+{ }_{j} I^{p}$ the representing $\omega$-category. It is equal to the direct limit of the diagram

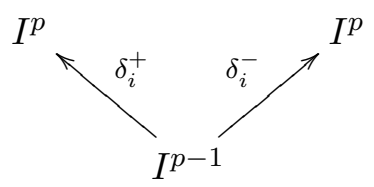

We denote by $\phi_{-}$and $\phi_{+}$the two canonical embeddings of $I^{p}$ in $I^{p}+{ }_{j} I^{p}$ respectively in the first and the second term.

Step 2. Using Yoneda, constructing a natural map

$$
+_{j}: \omega \operatorname{Cat}\left(I^{n}, \mathcal{C}\right) \times{ }_{j} \omega \operatorname{Cat}\left(I^{n}, \mathcal{C}\right) \longrightarrow \omega \operatorname{Cat}\left(I^{n}, \mathcal{C}\right)
$$


is equivalent to construct an $\omega$-functor $\eta_{n, j}$ from $I^{n}$ to $I^{n}+{ }_{j} I^{n}$ satisfying the dual properties. If $i<j$, then the natural transformation of functors $\left(\partial_{i}^{\alpha}, \partial_{i}^{\alpha}\right)$ yields an $\omega$-functor $\left(\delta_{i}^{\alpha}, \delta_{i}^{\alpha}\right)$ from $I^{n-1}+{ }_{j-1} I^{n-1}$ to $I^{n}+{ }_{j} I^{n}$. It is easy to see that this $\omega$-functor comes from the morphism of pasting scheme which associates to $\left(\beta, k_{1} \ldots k_{n-1}\right) \in I^{n-1}+_{j-1} I^{n-1}$ $\left(\beta, k_{1} \ldots[\alpha]_{i} \ldots k_{n-1}\right) \in I^{n}+{ }_{j} I^{n}$ with $\beta \in\{-,+\}$. If $i>j$, then the natural transformation of functors $\left(\partial_{i}^{\alpha}, \partial_{i}^{\alpha}\right)$ yields an $\omega$-functor $\left(\delta_{i}^{\alpha}, \delta_{i}^{\alpha}\right)$ from $I^{n-1}+{ }_{j} I^{n-1}$ to $I^{n}+{ }_{j} I^{n}$. It is easy to see that this $\omega$-functor comes from the morphism of pasting scheme which associates to $\left(\beta, k_{1} \ldots k_{n-1}\right) \in I^{n-1}+{ }_{j} I^{n-1}\left(\beta, k_{1} \ldots[\alpha]_{i} \ldots k_{n-1}\right) \in I^{n}+{ }_{j} I^{n}$ with $\beta \in\{-,+\}$. The properties which are required for the operations ${ }_{+j}$ entail the following relations for the $\eta_{n, j}$ :

$$
\begin{aligned}
& \eta_{n, j} \circ \delta_{j}^{ \pm}=\phi_{ \pm} \circ \delta_{j}^{ \pm} \\
& \eta_{n, j} \circ \delta_{i}^{\alpha}=\left(\delta_{i}^{\alpha}, \delta_{i}^{\alpha}\right) \circ \eta_{n-1, j-1} \text { if } i<j \\
& \eta_{n, j} \circ \delta_{i}^{\alpha}=\left(\delta_{i}^{\alpha}, \delta_{i}^{\alpha}\right) \circ \eta_{n-1, j} \text { if } i>j
\end{aligned}
$$

Step 3. The point is that it is difficult to find a formula for the composition of all cells of $I^{p}+{ }_{j} I^{p}$ (except in lower dimension). It is simpler to find this formula in a free $\omega$-category generated by a composable pasting scheme because composition means union in such a context Joh89. It turns out that $I^{p}+{ }_{j} I^{p}$ is exactly the free globular $\omega$-category generated by the composable pasting scheme defined as follows. Set

$$
\left(I^{p}+{ }_{j} I^{p}\right)_{q}=\left(\{-\} \times\left(I^{p}\right)_{q}\right) \cup\left(\{+\} \times\left(I^{p}\right)_{q}\right) / \equiv
$$

where $\equiv$ is the equivalence relation induced by the binary relation

$$
\left(-, k_{1} \ldots[+]_{j} \ldots k_{p-1}\right) \equiv\left(+, k_{1} \ldots[-]_{j} \ldots k_{p-1}\right)
$$

for every $k_{1}, \ldots, k_{p-1}$ in $\{-, 0,+\}$ together with the binary relations $E$ and $B$ defined by (with $x \in\left(I^{p}\right)_{i}$ and $y \in\left(I^{p}\right)_{j}$ )

$$
\begin{aligned}
& E_{j}^{i}=\left\{((a, x),(a, y)) \in\left(I^{p}+{ }_{j} I^{p}\right) \times\left(I^{p}+{ }_{j} I^{p}\right) / x E_{j}^{i} y \text { and } a \in\{-,+\}\right\} \\
& B_{j}^{i}=\left\{((a, x),(a, y)) \in\left(I^{p}+{ }_{j} I^{p}\right) \times\left(I^{p}+{ }_{j} I^{p}\right) / x B_{j}^{i} y \text { and } a \in\{-,+\}\right\}
\end{aligned}
$$

Now we are in position to prove the following property $P(n)$ by induction on $n$ : "for any $j$ between 1 and $n$, there exists one and only one $\omega$-functor $\eta_{n, j}$ from $I^{n}$ to $I^{n}+{ }_{j} I^{n}$ satisfying Condition 4, Condition 5, and Condition 6 ; moreover $\eta_{n, j}\left(R\left(0_{n}\right)\right)=R\left(\left\{\left(-, 0_{n}\right),\left(+, 0_{n}\right)\right\}\right.$ ". This latter equality illustrates the interest of globular pasting schemes.

Step 4. If $n=1$, we have to construct an $\omega$-functor from $I^{1}$ to $I^{1}+{ }_{1} I^{1}$. The hypotheses lead us to set $\eta_{1,1}(R(-))=R((-,-))$ and $\eta_{1,1}(R(+))=R((+,+))$. There exists thus one and only one suitable $\omega$-functor $\eta_{1,1}$ and this is the unique one which satisfies

$$
\eta_{1,1}(R(0))=R((-, 0)) *_{0} R((+, 0))=R((-, 0),(+, 0)) .
$$


So $P(1)$ is true. Suppose we have proved $P(k)$ for $k<n$ where $n$ is a natural number greater than 2 . We have to construct an $\omega$-functor $\eta_{n, j}$ for any $j$ between 1 and $n$ from $I^{n}$ to $I^{n}+{ }_{j} I^{n}$. The induction hypothesis and Condition 4 Condition 5 and Condition 6 entail the value of $\eta_{n, j}$ on all faces of $I^{n}$ of dimension at most $n-1$. It remains to prove that $\eta_{n, j}\left(R\left(0_{n}\right)\right)=R\left(\left\{\left(-, 0_{n}\right),\left(+, 0_{n}\right)\right\}\right)$ is one and the only solution. It suffices to verify that $s_{n-1} R\left(\left\{\left(-, 0_{n}\right),\left(+, 0_{n}\right)\right\}\right)=\eta_{n, j}\left(s_{n-1} R\left(0_{n}\right)\right)$ and that $t_{n-1} R\left(\left\{\left(-, 0_{n}\right),\left(+, 0_{n}\right)\right\}\right)=\eta_{n, j}\left(t_{n-1} R\left(0_{n}\right)\right)$. Let us verify the first equality. One has

$$
s_{n-1} R\left(0_{n}\right)=R\left(\delta_{1}^{(-)^{1}}\left(0_{n-1}\right), \ldots, \delta_{n}^{(-)^{n}}\left(0_{n-1}\right)\right)
$$

by the construction of $I^{n}$. By induction hypothesis, $\eta_{n, j}$ is $(n-1)$-extendable. Since composition means union, and because of Condition 4, Condition 5, and Condition 6, one has

$$
\begin{aligned}
& {\left[\bigcup_{h=1}^{h=j-1}\left(\delta_{h}^{(-)^{h}}, \delta_{h}^{(-)^{h}}\right) \circ \eta_{n-1, j-1}\left(0_{n-1}\right)\right] \cup\left(\phi_{-} \circ \delta_{j}^{(-)^{j}}\left(0_{n-1}\right)\right)} \\
& \cup\left[\bigcup_{h=j+1}^{h=n}\left(\delta_{h}^{(-)^{h}}, \delta_{h}^{(-)^{h}}\right) \circ \eta_{n-1, j}\left(0_{n-1}\right)\right] \\
& =\left[\bigcup_{h=1}^{h=j-1} R\left(\left(-, \delta_{h}^{(-)^{h}}\left(0_{n-1}\right)\right),\left(+, \delta_{h}^{(-)^{h}}\left(0_{n-1}\right)\right)\right)\right] \cup R\left(\left((-)^{j}, \delta_{j}^{(-)^{j}}\left(0_{n-1}\right)\right)\right) \\
& \cup\left[\bigcup_{h=j}^{h=n} R\left(\left(-, \delta_{h}^{(-)^{h}}\left(0_{n-1}\right)\right),\left(+, \delta_{h}^{(-)^{h}}\left(0_{n-1}\right)\right)\right)\right] \\
& \subset \eta_{n, j}\left(s_{n-1} R\left(0_{n}\right)\right)
\end{aligned}
$$

It suffices to verify that

$$
s_{n-1} R\left(\left(-, 0_{n}\right),\left(+, 0_{n}\right)\right)=R\left(\left\{\left((-)^{j}, \delta_{j}^{(-)^{j}}\left(0_{n-1}\right)\right),\left( \pm, \delta_{h}^{(-)^{h}}\left(0_{n-1}\right)\right) / h \neq j\right\}\right)
$$

in the pasting scheme $I^{n}+{ }_{j} I^{n}$ to complete the proof.

Let us define a natural map $\square_{n}$ from $\tau_{n} \mathcal{C}$ (the set of morphisms of $\mathcal{C}$ of dimension lower or equal than $n)$ to $\omega \operatorname{Cat}\left(I^{n}, \mathcal{C}\right)$ by induction on $n$ as follows. One sets $\square_{0}=\square_{0}^{-}$and $\square_{1}=\square_{1}^{-}$.

Proposition 10.2. For any natural number $n$ greater or equal than 2 , there exists a unique natural map $\square_{n}$ from $\mathcal{C}$ to $\omega$ Cat $\left(I^{n}, \mathcal{C}\right)$ such that

1. the equality $\square_{n}(x)\left(0_{n}\right)=x$ holds. 
2. one has $\partial_{1}^{\alpha} \square_{n}=\square_{n-1} d_{n-1}^{(-)^{\alpha}}$ for $\alpha= \pm$.

3. for $1<i \leqslant n$, one has $\partial_{i}^{\alpha} \square_{n}=\epsilon_{1} \partial_{i-1}^{\alpha} \square_{n-1} s_{n-1}$.

Moreover for $1 \leqslant i \leqslant n$, we have $\partial_{i}^{ \pm} \square_{n} s_{n} u=\partial_{i}^{ \pm} \square_{n} t_{n} u$ for any $(n+1)$-morphism $u$ and for all $u \in \tau_{n} \mathcal{C}, \square_{n}(u) \in \gamma \mathcal{N}^{\square}(\mathcal{C})_{n}$.

Proof. The induction equations define a fillable $(n-1)$-shell as defined in Proposition 5.1.

Proposition 10.3. For all $n \geq 0$, the evaluation map ev $v_{0_{n}}: x \mapsto x\left(0_{n}\right)$ from $\omega \operatorname{Cat}\left(I^{n}, \mathcal{C}\right)$ to $\mathcal{C}$ induces a bijection from $\gamma \mathcal{N}^{\square}(\mathcal{C})_{n}$ to $\tau_{n} \mathcal{C}$.

Proof. Obvious for $n=0$ and $n=1$. Let us suppose that $n \geq 2$ and let us proceed by induction on $n$. Since $e v_{0_{n}} \square_{n}(u)=u$ by the previous proposition, then the evaluation map $e v$ from $\gamma \mathcal{N}^{\square}(\mathcal{C})_{n}$ to $\tau_{n} \mathcal{C}$ is surjective. Now let us prove that $x \in \gamma \mathcal{N}^{\square}(\mathcal{C})_{n}$ and $y \in \gamma \mathcal{N} \square(\mathcal{C})_{n}$ and $x\left(0_{n}\right)=y\left(0_{n}\right)=u$ imply $x=y$. Since $x$ and $y$ are in $\gamma \mathcal{N} \square(\mathcal{C})_{n}$, then one sees immediately that the four elements $\partial_{1}^{ \pm} x$ and $\partial_{1}^{ \pm} y$ are in $\gamma \mathcal{N}^{\square}(\mathcal{C})_{n-1}$. Since all other $\partial_{i}^{\alpha} x$ and $\partial_{i}^{\alpha} y$ are thin, then $\partial_{1}^{-} x\left(0_{n-1}\right)=\partial_{1}^{-} y\left(0_{n-1}\right)=s_{n-1} u$ and $\partial_{1}^{+} x\left(0_{n-1}\right)=\partial_{1}^{+} y\left(0_{n-1}\right)=t_{n-1} u$. By induction hypothesis, $\partial_{1}^{-} x=\partial_{1}^{-} y=\square_{n-1}\left(s_{n-1} u\right)$ and $\partial_{1}^{+} x=\partial_{1}^{+} y=\square_{n-1}\left(t_{n-1} u\right)$. By hypothesis, one can set $\partial_{j}^{\alpha} x=\epsilon_{1}^{j-1} x_{j}^{\alpha}$ and $\partial_{j}^{\alpha} y=\epsilon_{1}^{j-1} y_{j}^{\alpha}$ for $2 \leqslant j \leqslant n$. And one gets $x_{j}^{\alpha}=\left(\partial_{1}^{\alpha}\right)^{j-1} \partial_{j}^{\alpha} x=\left(\partial_{1}^{\alpha}\right)^{j} x=\left(\partial_{1}^{\alpha}\right)^{j} y=y_{j}^{\alpha}$. Therefore $\partial_{j}^{\alpha} x=\partial_{j}^{\alpha} y$ for all $\alpha \in\{-,+\}$ and all $j \in[1, \ldots, n]$. By Proposition 5.1, one gets $x=y$.

The above proof also shows that the map which associates to any cube $x$ of the cubical singular nerve of $\mathcal{C}$ the cube $\square_{\operatorname{dim}(x)}\left(x\left(0_{\operatorname{dim}(x)}\right)\right)$ is exactly the usual folding operator as exposed in AA89.

Now let us remark that the free globular $\omega$-category generated by a cubical set $K$ can be also obtained by considering the image by the functor $\gamma$ of $\rho(K)$. Beware of the fact that in Al-Agl's $\mathrm{PhD}$, globular $\omega$-categories contain identity operators (his $\omega$-categories are $\mathbb{N}$ graded). So the correct statement is $(\gamma \rho(K))_{n}=\tau_{n} F(K)$ where $\tau_{n} F(K)$ is the $n$-category obtained by keeping only the $p$-morphisms with $p \leqslant n$. It suffices to prove the previous equality for $K=I^{n}$ since $\gamma$ is a left adjoint functor [AA89], therefore it commutes with all direct limits.

Corollary 10.4. Let $K$ be a cubical set. Then $\mathcal{N}^{\square}(F(K))$ is the free cubical $\omega$-category $\rho(K)$ generated by $K$.

Proof. By [AA89] Proposition 2.7.3, any $n$-cube $x$ of $\rho(K)$ (resp. of $\mathcal{N}^{\square}(F(K))$ ) is determined by its $(n-1)$-shell of $(n-1)$-faces $\left(\partial_{j}^{ \pm} x\right)_{1 \leqslant j \leqslant n+1}$ and by its image in $\gamma \rho(K)$ (resp. $\left.\gamma \mathcal{N}^{\square}(F(K))\right)$. 
Now take a cubical set $K$. As a consequence of the above remarks, it is possible to construct $H_{*}^{g l}(K)$ and $H_{*}^{ \pm}(K)$ and the two morphisms $h_{*}^{ \pm}$by using the free cubical $\omega$ category generated by $K$ instead of using the globular one. Let us still denote by $\gamma \rho(K)$ the globular $\omega$-category obtained by removing all identity elements. It is exactly the free globular $\omega$-category generated by $K$. We set $H_{*}^{g l}(K):=H_{*}^{g l}(\gamma \rho(K))$ and since $\mathcal{N}^{\square}(F(K))$ is the free cubical $\omega$-category generated by $K$, we set $H_{*}^{ \pm}(K)=H_{*}\left(\mathbb{Z} \rho(K)_{*}^{ \pm}, \partial^{ \pm}\right)$where

$$
\rho(K)_{n}^{ \pm}=\left\{x \in \rho(K)_{n}, \forall i_{1}, \ldots, i_{n-1}, \partial_{i_{1}}^{ \pm} \ldots \partial_{i_{n-1}}^{ \pm} x \in \rho(K)_{1}\right\} .
$$

The two morphisms $h_{*}^{ \pm}$from $H_{*}^{g l}(K)$ to $H_{*}^{ \pm}(K)$ are constructed like in Proposition 7.2 : the only tool to be used is again [AA89 Proposition 2.7.3.

\section{Acknowledgments}

Thanks are due to Eric Goubault for an uncalculable number of discussions about the geometry of automata, to Ronnie Brown and the referees for their helpful comments, to Emmanuel Peyre for the picture of the oriented 4-cube, and to Marc Wambst for his remarks about the redaction of the paper. 


\section{The categories and functors of this work}

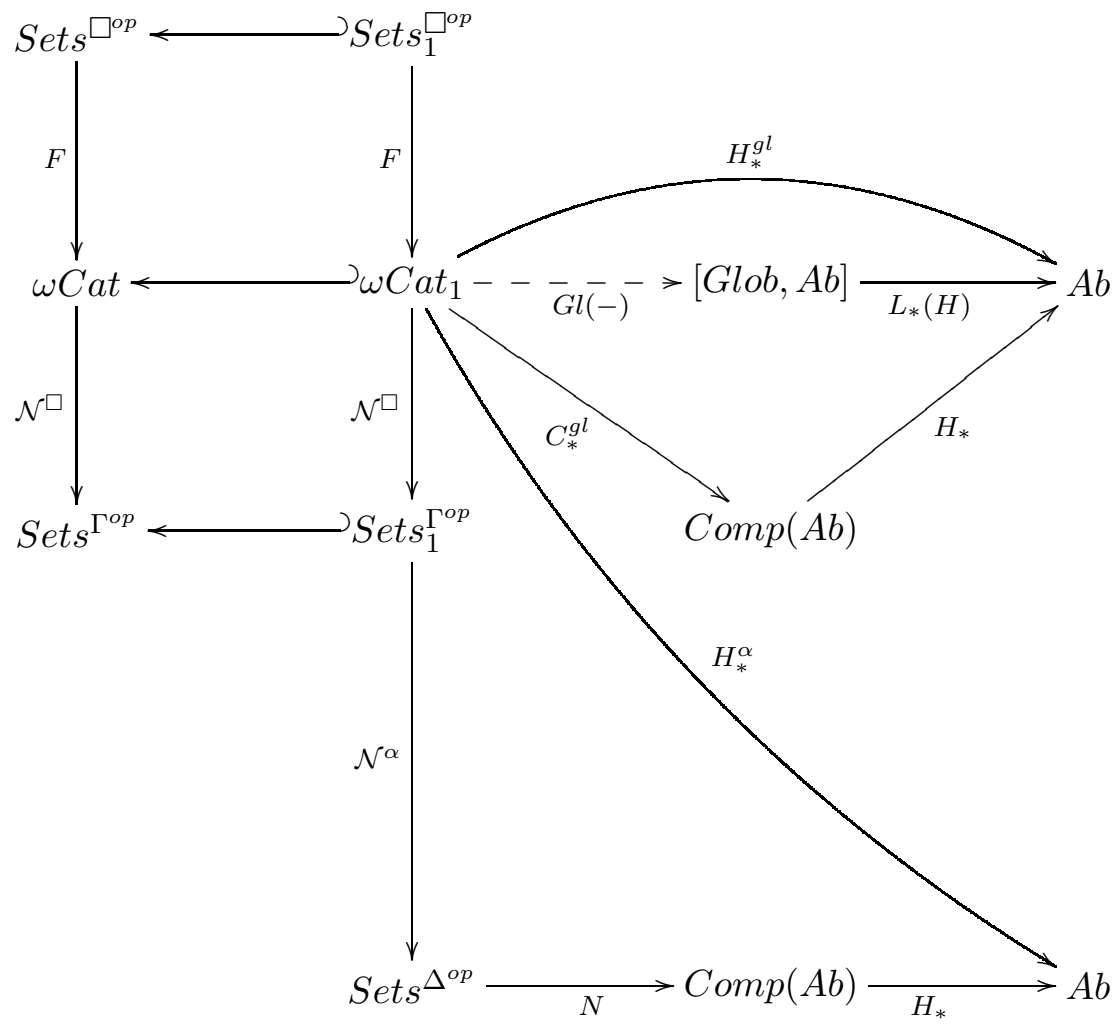

The above diagram is commutative in the sense that two different ways between the same pair of points give the same transformation. All these transformations are functors except $G l(-)$. This diagram summarizes all transformations or functors constructed in this paper.

\section{List of Figures}

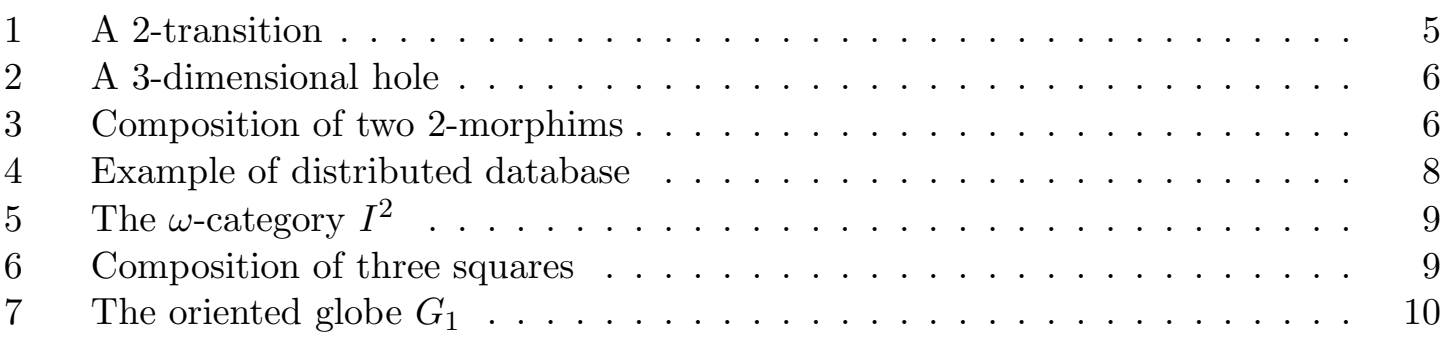


$8 \quad$ The Swiss Flag . . . . . . . . . . . . . . . . . . . . . . . . . 10

9 Unsafe area and unreachable area in a concurrent machine with semaphores 12

$10 \quad$ A loop which does not give rise to a globular cycle . . . . . . . . . . . . . . . 12

11 Example of globular cycle in higher dimension . . . . . . . . . . . . . . 13

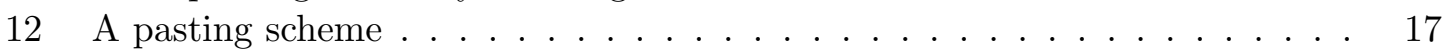

13 The $\omega$-category $I^{3} \ldots \ldots \ldots \ldots \ldots \ldots \ldots$

14 The $\omega$-category $I^{4} \ldots \ldots \ldots \ldots \ldots$

15 A 1-dimensional negative corner . . . . . . . . . . . . . . . . 22

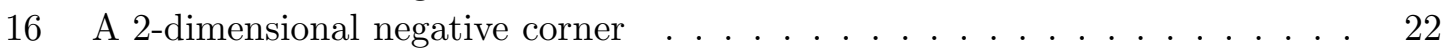

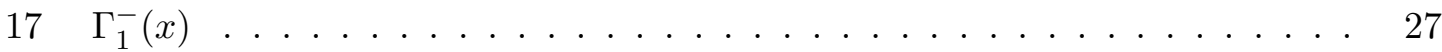

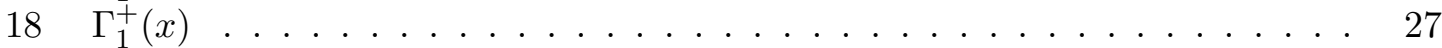

19 A 2-semaphor $\ldots \ldots \ldots \ldots$. . . . . . . . . . . . . . . . . . . . 39

20 Composition of two 2-morphims . . . . . . . . . . . . . . . . . . 40

21 Two 0-composable 2-morphisms . . . . . . . . . . . . . . . . . 40

$22 h_{2}^{-}\left(A *_{1} B-A-B\right)$ is a boundary $\ldots \ldots \ldots \ldots \ldots . \ldots \ldots$

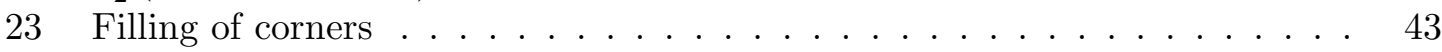

\section{References}

[AA89] Fahd Ali A. Al-Agl. Aspects of multiple categories. PhD thesis, University of Wales, Department of Pure Mathematics, University College of North Wales, Bangor, Gwynedd LL57 1UT, U.K., September 1989.

[Ait86] Iain R. Aitchison. The geometry of oriented cubes. Macquarie Mathematics Reports 86-0082, 1986.

[ARJ86] A.V.Aho, R.Sethi, and J.D.Ullman. Compilers, principle, techniques and tools. Addison-Wesley, 1986.

[Bat98] M.A. Batanin. Monoidal globular categories as a natural environment for the theory of weak n-categories. Advances in Mathematics, 136(1):39-103, 1998.

[BH81a] R. Brown and P.-J. Higgins. The equivalence of $\infty$-groupoids and crossed complexes. Cahier de Topologie et de Géométrie différentielle, 22:371-386, 1981.

[BH81b] R. Brown and P.-J. Higgins. On the algebra of cubes. Journal of Pure and Applied Algebra, 21:233-260, 1981.

[BM99] R. Brown and G.-H. Mosa. Double categories, 2-categories, thin structures and connections. Theory and Application of Categories, 5:163-175, 1999. 
[Bor94] Francis Borceux. Handbook of categorical algebra, volume 50. Cambridge University Press, 1994.

[Cra95] Sjoerd E. Crans. Pasting schemes for the monoidal biclosed structure on wcat. Utrecht University, April 1995.

[Dij68] E.W. Dijkstra. Cooperating Sequential Processes. Academic Press, 1968.

[FGR98a] L. Fajstrup, E. Goubault, and M. Raussen. Algebraic topology and concurrency. preprint, 1998.

[FGR98b] L. Fajstrup, E. Goubault, and M. Raussen. Detecting deadlocks in concurrent systems. In D. Sangiorgi R. de Simone, editor, CONCUR '98; Concurrency Theory, number 1466 in Lecture Notes in Computer Science, pages 332-347. Springer-Verlag, September 1998.

[Gau97a] Philippe Gaucher. Confidentialité en dimension 1, 1997.

[Gau97b] Philippe Gaucher. Entrée et sortie d'automates en algèbre homologique. Technical report, IRMA, 1997.

[Gau97c] Philippe Gaucher. Etude homologique des chemins de dimension 1 dans un automate. Technical report, IRMA, 1997.

[Gou95] Eric Goubault. The Geometry of Concurrency. PhD thesis, Ecole Normale Supérieure, 1995.

[GW91] P. Godefroid and P. Wolper. Using partial orders for the efficient verification of deadlock freedom and safety properties. In Proc. of the Third Workshop on Computer Aided Verification, volume 575, pages 417-428. Springer-Verlag, Lecture Notes in Computer Science, July 1991.

[HR94] M. Herlihy and S. Rajsbaum. Set consensus using arbitrary objects. In Proc. of the 13th Annual ACM Symposium on Principles of Distributed Computing. ACM Press, August 1994.

[HS94] M. Herlihy and N. Shavit. A simple constructive computability theorem for wait-free computation. In Proceedings of STOC'94. ACM Press, 1994.

[Joh89] M. Johnson. The combinatorics of $n$-categorical pasting. Journal of Pure and Applied Algebra, 62:211-225, 1989.

[KP97] K.H. Kamps and T. Porter. Abstract Homotopy and Simple Homotopy Theory. World Scientific, 1997. 
[KV91] M.M. Kapranov and V.A. Voedvodsky. Combinatorial-geometric aspects of polycategory theory : pasting schemes and higher bruhat orders. Cahiers de topologie et gomtrie diffrentielle catgoriques, XXXII-1:11-26, 1991.

[May67] J. Peter May. Simplicial Objects in Algebraic Topology. D. Van Nostrand Company, 1967.

[ML71] Saunders Mac-Lane. Categories for the Working Mathematician, volume 5 of Graduate Texts in Mathematics. Springer-Verlag, 1971.

[Pen99] Jacques Penon. Approche polygraphique des $\infty$-catégories non strictes. Cahiers de topologie et géométrie différentielle catégoriques, XL-1, 1999.

[Pra91] V. Pratt. Modeling concurrency with geometry. In ACM Press, editor, Proc. of the 18th ACM Symposium on Principles of Programming Languages, 1991.

[Rot79] Joseph J. Rotman. An Introduction to Homological Algebra. Academic Press, 1979.

[Sok99] Stefan Sokolowski. Investigation of concurrent processes by means of homotopy functors. 45 pages, June 1999.

[Spe77] C.B. Spencer. An abstract setting for homotopy pushouts and pullbacks. Cahier de topologie et gomtrie diffrentielle catgorique, 18:409-430, 1977.

[Ste91] Richard Steiner. Tensor products of infinity-categories. University of Glasgow, 1991.

[Str87] Ross Street. The algebra of oriented simplexes. Journal of Pure and Applied Algebra, 49:283-335, 1987.

[Str98] Ross Street. The petit topos of globular sets. Macquarie Mathematics Report 98/232, 1998.

[SW83] C.B. Spencer and Y.L. Wong. Pullback and pushout squares in a special double category with connections. Cahier de topologie et gomtrie diffrentielle catgorique, 18:161-192, 1983.

[vG91] R. van Glabbeek. Bisimulation semantics for higher dimensional automata. Technical report, Stanford University, 1991.

[Wei94] C.A. Weibel. An introduction to homological algebra. 38. Cambridge studies in advanced mathematics, 1994.

[Yos83] T. Yoshida. Projective $\gamma$-sets. Hokkaido Mathematical Journal, pages 10-16, 1983. 